\title{
Predicting alloy vibrational mode properties using lattice dynamics calculations, molecular dynamics simulations, and the virtual crystal approximation
}

\author{
Jason M. Larkin and Alan J. H. McGaugheya) \\ Department of Mechanical Engineering, Carnegie Mellon University, Pittsburgh, Pennsylvania 15213, USA
}

(Received 26 April 2013; accepted 17 June 2013; published online 9 July 2013)

\begin{abstract}
The virtual crystal (VC) approximation for mass disorder is evaluated by examining two model alloy systems: Lennard-Jones argon and Stillinger-Weber silicon. In both material systems, the perfect crystal is alloyed with a heavier mass species up to equal concentration. The analysis is performed using molecular dynamics simulations and lattice dynamics calculations. Mode frequencies and lifetimes are first calculated by treating the disorder explicitly and under the VC approximation, with differences found in the high-concentration alloys at high frequencies. Notably, the lifetimes of high-frequency modes are underpredicted using the $\mathrm{VC}$ approximation, a result we attribute to the neglect of higher-order terms in the model used to include point-defect scattering. The mode properties are then used to predict thermal conductivity under the $\mathrm{VC}$ approximation. For the Lennard-Jones alloys, where high-frequency modes make a significant contribution to thermal conductivity, the high-frequency lifetime underprediction leads to an underprediction of thermal conductivity compared to predictions from the Green-Kubo method, where no assumptions about the thermal transport are required. Based on observations of a minimum mode diffusivity, we propose a correction that brings the $\mathrm{VC}$ approximation thermal conductivities into better agreement with the Green-Kubo values. For the Stillinger-Weber alloys, where the thermal conductivity is dominated by low-frequency modes, the high-frequency lifetime underprediction does not affect the thermal conductivity prediction and reasonable agreement is found with the Green-Kubo values. (C) 2013 AIP Publishing LLC. [http://dx.doi.org/10.1063/1.4812737]
\end{abstract}

\section{INTRODUCTION}

Due to their potentially low thermal conductivities, disordered materials (e.g., alloys, amorphous solids, aerogels) are used in applications ranging from thermoelectric energy conversion to thermally insulating barriers. ${ }^{1-10}$ Disordered lattices are a subgroup of disordered materials where the atomic positions follow a lattice structure but the constituent species are spatially random. Examples include isotopic solids, where the species have the same electronic structure but small mass variations, ${ }^{11,12}$ and alloys, our focus here, where at least two distinct species are present. ${ }^{13,14}$

We further restrict our focus to dielectric or semiconducting solids, where the heat is conducted by the atomic vibrational modes. Predicting the thermal conductivity of such materials requires the properties of the full spectrum of vibrational modes. ${ }^{15-17}$ Accurate predictions of these properties for crystalline systems (i.e., perfect lattices) can be made with anharmonic lattice dynamics (ALD) theory using input from density functional theory (DFT) calculations. ${ }^{12,18-26}$ Computational costs limit DFT calculations to less than 100 atoms, however, making it challenging to explicitly incorporate the effects of disorder. ${ }^{12,20,22,25,27-29}$

Disorder is typically included in the ALD framework using Abeles' virtual crystal (VC) approximation, whereby the disordered solid is replaced with a perfect VC with properties equivalent to an averaging over the disorder (e.g.,

${ }^{\text {a)} E l e c t r o n i c ~ a d d r e s s: ~ m c g a u g h e y @ c m u . e d u ~}$ atomic mass and/or bond strength). ${ }^{14}$ The ALD calculations are performed on a small unit cell with the averaged properties (i.e., all vibrational modes are phonons) and phononphonon and phonon-disorder scattering are included as perturbations. ${ }^{11,12,14,20,22}$ Except for low-frequency (long-wavelength) acoustic modes, the general validity of this assumption is unclear. We will refer to this approach as VCALD. Recent work using DFT calculations and the VC-ALD approach has modeled disordered lattices with relatively large $(\sim 10-100 \mathrm{~W} / \mathrm{m}-\mathrm{K})^{12,20,25}$ and small $(\sim 1 \mathrm{~W} / \mathrm{m}-\mathrm{K})^{22}$ thermal conductivities. No comprehensive study has been performed to assess the applicability of the VC-ALD approach for a range of disorder strength.

The objective of this study is to investigate the use of the $\mathrm{VC}$ approximation for predicting the vibrational mode properties and thermal conductivities of alloys by a detailed comparison of three predictive methods: (i) molecular dynamics (MD)-based normal mode decomposition (NMD), (ii) MDbased Green-Kubo (GK), and (iii) VC-ALD. By using computationally inexpensive empirical potentials for argon [Lennard-Jones (LJ) at a temperature of $10 \mathrm{~K}]^{30}$ and silicon [Stillinger-Weber (SW) at a temperature of $300 \mathrm{~K}],{ }^{31}$ we can self-consistently study the effects of disorder both explicitly and as a perturbation. For both materials, the perfect lattice is disordered with a heavier mass species up to equal concentration, spanning a range of small to large disorder. By spanning this range, the limits of the perturbative models are examined.

The remainder of the paper is organized as follows. In Sec. II, the theoretical formulation of thermal transport in 
ordered and disordered solids and the computational framework are described. In Sec. III, the frequencies, group velocities, lifetimes, and diffusivities of the vibrational modes of the LJ argon alloys are predicted when the disorder is explicitly modeled and when it is treated as a perturbation in the VC approximation. A breakdown of the VC-ALD method is identified by a comparison with the VC-NMD method in Sec. III C 2 and a correction is suggested in Sec. IIID. The vibrational mode properties are then used to predict thermal conductivities in Sec. IV, allowing for a comparison to the predictions of the top-down GK method, where no assumptions about the nature of the thermal transport are required. The vibrational mode properties and thermal conductivity of the SW silicon alloys, where low-frequency modes dominate the thermal conductivity, are predicted in Sec. $\mathrm{V}$ to provide a comparison and contrast to the LJ argon alloys.

\section{THEORETICAL AND COMPUTATIONAL FRAMEWORK}

\section{A. Thermal conductivity prediction}

To predict the thermal conductivity of a disordered lattice, one begins with the theory for a perfect lattice. For a perfect lattice, all vibrational modes are phonon modes, which by definition are delocalized, propagating plane waves. ${ }^{15}$ Using the single-mode relaxation time approximation $^{15}$ to solve the Boltzmann transport equation gives an expression for thermal conductivity in direction $\mathbf{n}$,

$$
k_{p h, \mathbf{n}}=\sum_{\boldsymbol{\kappa}} \sum_{\nu} c_{p h}\left(\begin{array}{l}
\boldsymbol{\kappa} \\
\nu
\end{array}\right) v_{g, \mathbf{n}}^{2}\left(\begin{array}{l}
\boldsymbol{\kappa} \\
\nu
\end{array}\right) \tau\left(\begin{array}{l}
\boldsymbol{\kappa} \\
\nu
\end{array}\right) .
$$

Here, the sum is over the phonon modes in the first Brillouin zone, $\boldsymbol{\kappa}$ is the wave vector, and $\nu$ labels the polarization branch. The phonon mode has frequency $\omega\left(\begin{array}{l}\boldsymbol{\kappa} \\ \nu\end{array}\right)$, volumetric specific heat $c_{p h}\left(\begin{array}{l}\boldsymbol{\kappa} \\ \nu\end{array}\right)$, n-component of the group velocity vector $v_{g, \mathbf{n}}\left(\begin{array}{l}\boldsymbol{\kappa} \\ \nu\end{array}\right)$, and lifetime $\tau\left(\begin{array}{l}\boldsymbol{\kappa} \\ \nu\end{array}\right)$.

The relaxation time approximation has been found to be valid for lower thermal conductivity materials (e.g., Si and SiGe alloys), ${ }^{18-20}$ while larger thermal conductivity materials such as $\mathrm{GaN}$ and diamond require an iterative solution to the Boltzmann transport equation (BTE) for more accurate predictions using Eq. (1). ${ }^{12,27}$ For the crystalline LJ argon and SW silicon phases, the lattices and the components of their thermal conductivity tensors are cubically symmetric, so that we will refer to $k_{p h}$ as an isotropic scalar thermal conductivity. This isotropy will hold for disordered lattices in the infinite-size limit. Since MD simulations are classical and obey Maxwell-Boltzmann statistics, ${ }^{32}$ the volumetric specific heat is $k_{\mathrm{B}} / V$ per mode in the harmonic limit, where $V$ is the system volume and $k_{\mathrm{B}}$ is the Boltmann constant. This harmonic approximation for specific heat has been shown to be valid for LJ argon and SW silicon at the temperatures of interest here $\mathrm{r}^{33,34}$ and is used so that direct comparisons can be made between the MD- and lattice dynamics-based methods.

For disordered systems, the vibrational modes are no longer pure plane-waves (i.e., phonon modes), except in the low-frequency (long-wavelength) limit. When applied in the classical limit, the Allen-Feldman (AF) theory computes the contribution of diffusive, non-propagating modes (i.e., diffusion(s)) to thermal conductivity from ${ }^{35}$

$$
k_{A F}=\sum_{\text {diffusons }} \frac{k_{\mathrm{B}}}{V} D_{A F, i}\left(\omega_{i}\right)
$$

where $D_{A F, i}$ is the mode diffusivity and $\omega_{i}$ is the frequency of the $i$ th diffusion. The diffusivity of diffusion(s) can be calculated from harmonic lattice dynamics theory. ${ }^{16,35,36}$

Assuming that all vibrational modes travel with the sound speed, $v_{s}$, and scatter over a distance of the lattice constant, $a$, a high-scatter (HS) limit of thermal conductivity in the classical limit is ${ }^{2}$

$$
k_{H S}=\frac{k_{\mathrm{B}}}{V_{b}} b v_{s} a
$$

where $V_{b}$ is the volume of the unit cell and $b$ is the number of atoms in the unit cell. The HS limit will be used to discuss the differences between the LJ argon and SW silicon alloys.

\section{B. Virtual crystal approximation}

Under the VC approximation, the disordered solid is replaced with a perfect, single-species crystal with properties (e.g., density and cohesive energy) equivalent to an averaging over the disorder (e.g., atomic mass and/or bond strength). ${ }^{14}$ The VC approximation is visualized for an alloy in Figs. 1(a) and 1(b), where a mass-disordered supercell is replaced by a perfect crystal with an averaged mass. Abeles first introduced the concept of a VC to predict the thermal conductivity of SiGe, GaAs/InAs, and InAs/InP alloys. ${ }^{14}$ Klemens-Callaway theory, which is valid for low-frequency modes and small disorder, was used to model the phononphonon and phonon-defect scattering. ${ }^{14,37-41}$ The Abeles theory is conceptually simple, treating both disorder and anharmonicity as perturbations, and leads to a closed-form analytical function for the thermal conductivity. With the use of phenomenological fitting parameters, good agreement between the predictions and experimental measurements was found for SiGe and GaAs/InAs alloys. Deviations were observed for InAs/InP alloys at large concentrations of InP, which were attributed to the large mass ratio of 3.7 between indium and phosphorus. ${ }^{14}$

When considering alloys, it is important to note that the overall disorder strength is determined by the mass ratio, the stiffness ratio, and the alloy concentration. Cahill and coworkers found that as little as $6.2 \times 10^{19} \mathrm{~cm}^{-3}$ germanium reduces the thermal conductivity of epitaxial silicon layers by a factor of two. ${ }^{42}$ Using the Abeles theory, they explained this result by mass perturbative disorder alone (the $\mathrm{Ge} / \mathrm{Si}$ mass ratio is 2.6). ${ }^{42,43}$ The relative effects of bond and mass disorder were investigated computationally using MD 
(a) Disordered Supercell

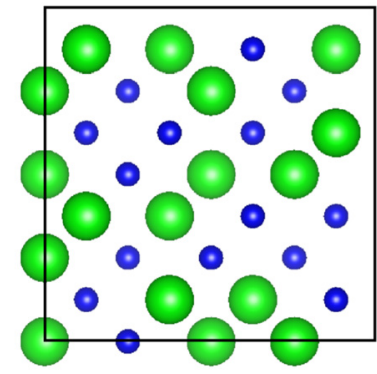

(b) VC Unit Cell

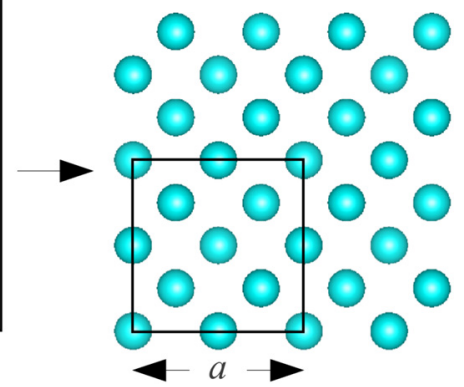

FIG. 1. (a) Explicitly disordered alloy supercell of silicon and "heavy" silicon ([100] direction into the page).$^{50}$ (b) Equivalent VC supercell with one averaged mass. The sphere size represents increasing mass only, no bond disorder is considered. The 8-atom conventional cubic unit cell is shown in (b).

simulations by Skye and Schelling for SiGe alloys up to equal concentration. ${ }^{44}$ They also found that mass disorder is the dominant scattering mechanism. Subsequent studies have modeled the effect of differing species by only including atomic mass differences. ${ }^{45,46}$

Unlike the phenomenological Abeles theory, the VCALD approach predicts thermal conductivity by directly summing over the modes of the full vibrational spectrum, with phonon-phonon and phonon-defect scattering treated as perturbations. ${ }^{12,20,22}$ In the VC-ALD method, the phononphonon scattering is predicted using ALD. ${ }^{24,47}$ The phonondefect scattering is treated using perturbative methods that can handle mass and/or bond disorder. ${ }^{11,37,38,40}$ In $\mathrm{Ni}_{0.55} \mathrm{Pd}_{0.45}$, which has a large mass ratio (1.8) and concentration of each species, experimental measurements of vibrational frequencies and linewidths agree well with predictions from the perturbative mass-disorder theory. ${ }^{11,40,41}$

Using DFT methods to predict the mode-specific phonon properties of the VC, Lindsay and Broido found good agreement between VC-ALD and experimental measurements of thermal conductivity for isotopically defected GaN (the gallium isotopes have concentrations of 0.6 and 0.4 and a mass ratio of 1.03). ${ }^{12}$ Garg et al. used DFT calculations with VC-ALD to predict the thermal conductivity of SiGe alloys for all concentrations at a temperature of $300 \mathrm{~K}$, obtaining good agreement with experiment. ${ }^{20}$ By including disorder explicitly in their ALD calculations, the predicted thermal conductivity decreased by $15 \%$. Isotopically defected $\mathrm{GaN}$ and low concentration SiGe alloys have relatively large thermal conductivities at a temperature of $300 \mathrm{~K}(\sim 100 \mathrm{~W} / \mathrm{m}-\mathrm{K})$. Li et al. used DFT calculations with VC-ALD to predict the thermal conductivity of $\mathrm{Mg}_{2} \mathrm{Si}_{x} \mathrm{Sn}_{1-x}(\sim 10 \mathrm{~W} / \mathrm{m}-\mathrm{K})$ in good agreement with experimental measurements for all concentrations. $^{25}$ The VC-ALD approach has also been used to predict the effect of interfacial mixing in GaAs/AlAs superlattices, but the thermal conductivity predictions were not compared with experimental measurements. ${ }^{26}$ In our survey of experimental measurements and numerical modeling, we find that VC predictions tend to be accurate when the disordered lattice thermal conductivity is significantly above the high-scatter limit [Eq. (3)], which tends to be around $1 \mathrm{~W} / \mathrm{m}-\mathrm{K} .^{2,12,14,20,41-43}$
An ALD study using phonon properties from DFT calculations for crystalline $\mathrm{PbTe}^{21}$ predicted thermal conductivities of $2 \mathrm{~W} / \mathrm{m}-\mathrm{K}$ at a temperature of $300 \mathrm{~K}$ in fair agreement with experiment. For PbTeSe alloys, a VC-ALD study predicted a small thermal conductivity reduction compared to the perfect crystals. ${ }^{22}$ Experimental results are limited for these alloys, ${ }^{48,49}$ making it difficult to asses the validity of the VC-ALD approach for materials whose thermal conductivities approach the high-scatter limit.

Given all these results, it is unclear what limitations exist for using the VC approach. In this study, we will consider a low thermal conductivity alloy using the LJ potential and a high thermal conductivity alloy using the SW potential. The computational studies discussed above were limited to VC-ALD because of DFT calculation costs. Our use of computationally inexpensive empirical potentials allows us to include the disorder explicitly and as a perturbation and to compare the predictions.

\section{Calculation and simulation details}

The key to explicitly incorporating the effects of disorder is to use large disordered supercells. Perfect and disordered lattice supercells are generated using the conventional unit cells for LJ argon $(n=4)$ and SW silicon $(n=8)$, where $n$ is the number of atoms in the unit cell. Supercells are built cubically with size $N_{0}$, where $N_{0}$ is the number of unit cell repetitions in the three spatial directions. Supercells up to $N_{0}=12$ (6096 atoms) are used for the LJ argon calculations. For SW silicon, $N_{0}=8$ (4096 atoms) is used for the MD-based NMD calculations and $N_{0} \leq 42$ (592704 atoms) is used for the MD-based GK and VC-ALD.

Disorder is created by randomly specifying the masses of the atoms on the lattice. The composition of each lattice is labeled by $m_{1-c}^{i} m_{c}^{j}$, where (i) $m^{i}=1$ and $m^{j}=3$ in LJ units for argon, and (ii) $m^{i}=m_{S i}$ and $m^{j}=2.6 m_{S i}$ for SW silicon and "heavy silicon," which has the mass of germanium. Concentrations, $c$, of $0,0.05,0.15$, and 0.5 are considered.

For LJ argon, the lattice constant at a temperature of $10 \mathrm{~K}$ is $5.290 \AA^{51}$ The MD simulations were performed using LAMMPS. ${ }^{52}$ Efficient MD codes like LAMMPS scale linearly with the number of atoms in the system, $N_{a}$, which makes the GK method (see Sec. IV) computationally inexpensive when used with empirical potentials. An amorphous LJ phase, discussed in Sec. III D, was created by liquefying the crystal and instantly quenching by removing all kinetic energy. The energy of the resulting structure was minimized and then annealed in an NPT (constant number of atoms $N$, pressure $P$, and temperature $T$ ) ensemble at zero pressure and a temperature of $10 \mathrm{~K}$. The effective zero-pressure lattice constant of the amorphous phase at this temperature, based on the atomic density, is $5.389 \AA$. For SW silicon, we use a lattice constant of $5.43 \AA$ for all calculations, which brings the perfect crystal GK thermal conductivity predictions at a temperature of $300 \mathrm{~K}$ (Refs. 34 and 53) into better agreement with ALD predictions ${ }^{54}$ compared to using the zero-pressure lattice constant.

All MD simulations are first equilibrated in a NVT (constant number of atoms, volume, and temperature) ensemble 
for $10^{6}$ time steps. Data are then collected from simulations in the NVE (constant number of atoms, volume, and total energy) ensemble. For LJ argon, the potential energy is cutoff and shifted at $8.5 \AA$ (the force is not adjusted). Time steps of 4.285 and 0.5 fs were used for the LJ argon and SW silicon simulations. The same atomic trajectories are used for the NMD and GK methods.

\section{VIBRATIONAL MODE PROPERTIES IN ALLOYS}

\section{A. Density of states (DOS)}

In this section, we begin to examine the effects of explicitly including disorder by computing the frequencies and DOS for the vibrational modes of disordered LJ lattice supercells and their equivalent VCs. The frequencies are computed using harmonic lattice dynamics calculations with GULP. ${ }^{55}$ For the VC, the allowed wave vectors are set by $N_{0}$ and, due to the use of the conventional unit cell, there are 12 polarization branches per wave vector. For the disordered supercells (referred to herein as Gamma), the only allowed wave vector is the gamma-point (i.e., $\boldsymbol{\kappa}=0$ ), where there are $12 N_{0}^{3}$ polarization branches. Calculation of the Gamma modes require the eigenvalue solution of a dynamical matrix of size $\left(3 N_{a}\right)^{2}$ that scales as $\left[\left(3 N_{a}\right)^{2}\right]^{3}$, limiting the system sizes that can be considered. This eigenvalue solution is also required to perform the Gamma-NMD (see Sec. III C 1) and AF calculations (see Sec. III D).

The DOS for the VC and Gamma modes are plotted in Figs. 2(a)-2(c) for concentrations of $0.05,0.15$, and 0.5 for $N_{0}=12$ (6912 atoms). The VC and Gamma DOS agree at low frequencies for all concentrations, where they follow the prediction of the Debye approximation that the DOS will scale as $\omega^{2} .{ }^{30}$ Similar agreement between VC and Gamma DOS at low frequencies was found in DFT predictions for $\mathrm{Si}_{c} \mathrm{Ge}_{1-c}$ (Ref. 20) and classical models of amorphous $\mathrm{Si}_{c} \mathrm{Ge}_{1-c}{ }^{56}$ The Debye approximation underpredicts the DOS at moderate frequency, which is due to non-linearities in the dispersion, ${ }^{30}$ but the VC and Gamma predictions remain in good agreement.

The increasing average atomic mass with increasing concentration for the VC shifts all frequencies downward by a factor $1 /\left[(1-c) m^{i}+c m^{j}\right]^{1 / 2}$. The increasing average atomic mass for the Gamma modes also reduces the frequencies, but not in a systematic manner. The effect of the disorder is seen at frequencies greater than ten by a broadening and shift of the Gamma DOS to higher frequencies because of the explicit use of light atoms in the supercell. This effect becomes more pronounced as the concentration increases. Duda et al. observed similar high-frequency broadening effects in model LJ alloys. ${ }^{57}$ The high-frequency broadening is an indication of phonon localization, which is known to first occur at the Brillouin zone edge. ${ }^{58}$ Based on the DOS, the vibrational modes of the explicitly disordered supercells at low frequencies are propagating, while the broadening of the DOS at high-frequency indicates that the Gamma vibrational modes may differ from the VC phonon modes in this regime. This behavior is further investigated in the next three sections.

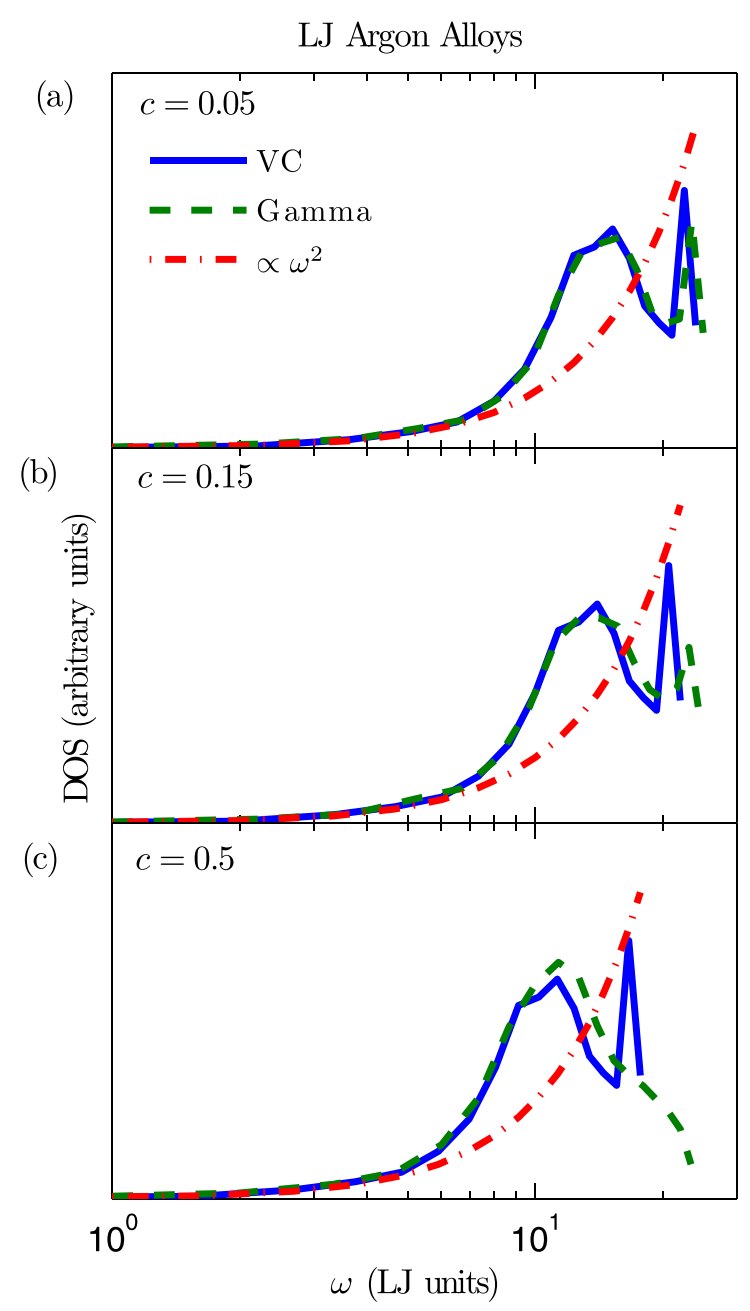

FIG. 2. Vibrational DOS for LJ alloys calculated using the VC approximation and an explicitly disordered supercell (labeled Gamma) for concentrations of (a) 0.05, (b) 0.15, and (c) 0.5. VC and Gamma show similar low-frequency behavior for all concentrations. For increasing concentrations, the frequencies of both VC and Gamma decrease, while the high frequency DOS for Gamma spreads and reaches to a higher maximum frequency because of the explicit disorder. The supercells are of size $N_{0}=12$ (6912 atoms).

\section{B. Dispersion and group velocity}

The group velocity vector in a VC is defined as the gradient of the dispersion curve

$$
\mathbf{v}_{g, \mathbf{n}}\left(\begin{array}{l}
\boldsymbol{\kappa} \\
\nu
\end{array}\right)=\frac{\partial \omega\left(\begin{array}{l}
\boldsymbol{\kappa} \\
\nu
\end{array}\right)}{\partial \boldsymbol{\kappa}} .
$$

We calculate the group velocities for the VC using finite differences on the frequencies calculated from harmonic lattice dynamics. ${ }^{59}$

For a disordered solid, the three acoustic group velocities (two transverse and one longitudinal) can be predicted using the elastic constants ${ }^{55}$ or by finite differencing of the three lowest frequency branches of the dispersion relation of the supercell. ${ }^{60,61}$ Except for this low-frequency behavior, there is not an accepted method to predict the group velocity of a vibrational mode in a disordered system, although there have been attempts. ${ }^{2,57,60-63}$ In the Cahill-Pohl model, for 
example, the group velocity of all disordered modes is the sound speed, $v_{s}$, which is also assumed for the high-scatter model, Eq. (3). ${ }^{2}$ This assumption is not generally valid for any material. ${ }^{36,57,60-63,65}$

Calculating the structure factors of the supercell modes is a method to test for their plane-wave character at a particular wave vector and polarization corresponding to the VC. ${ }^{17,36}$ Feldman et al. used the structure factor to predict an effective dispersion for a model of amorphous silicon, but did not predict group velocities. ${ }^{36}$ Volz and Chen used the dynamic structure factor to predict the dispersion of crystalline SW silicon using MD simulation. ${ }^{64}$ Recently, the effective dispersion of a model disordered lattice was predicted using the structure factor. ${ }^{65}$ as $^{17}$

The structure factor at a VC wave vector $\boldsymbol{\kappa}_{V C}$ is defined

$$
S^{L, T}\left(\begin{array}{c}
\boldsymbol{\kappa}_{V C} \\
\omega
\end{array}\right)=\sum_{\nu} E^{L, T}\left(\begin{array}{c}
\boldsymbol{\kappa}_{V C} \\
\nu
\end{array}\right) \delta\left[\omega-\omega\left(\begin{array}{c}
\boldsymbol{\kappa}=\mathbf{0} \\
\nu
\end{array}\right)\right],
$$

where the summation is over the Gamma modes, $E^{T}$ refers to the transverse polarization and is defined as

$E^{L}\left(\begin{array}{c}\boldsymbol{\kappa}_{V C} \\ \nu\end{array}\right)=\left|\sum_{b} \hat{\boldsymbol{\kappa}}_{V C} \cdot e\left(\begin{array}{cc}\boldsymbol{\kappa}=\mathbf{0} & b \\ \nu & \alpha\end{array}\right) \exp \left[i \boldsymbol{\kappa}_{V C} \cdot \boldsymbol{r}_{0}\left(\begin{array}{c}l=0 \\ b\end{array}\right)\right]\right|^{2}$

and $E^{L}$ refers to the longitudinal polarization and is defined as

$E^{T}\left(\begin{array}{c}\boldsymbol{\kappa}_{V C} \\ \nu\end{array}\right)=\left|\sum_{b} \hat{\boldsymbol{\kappa}}_{V C} \times e\left(\begin{array}{cc}\boldsymbol{\kappa}=\mathbf{0} & b \\ \nu & \alpha\end{array}\right) \exp \left[i \boldsymbol{\kappa}_{V C} \cdot \boldsymbol{r}_{0}\left(\begin{array}{c}l=0 \\ b\end{array}\right)\right]\right|^{2}$.

In Eqs. (6) and (7), the $b$ summations are over the atoms in the disordered supercell, $\boldsymbol{r}_{0}\left(\begin{array}{c}l=0 \\ b\end{array}\right)$ refers to the equilibrium atomic position of atom $b$ in the supercell, $l$ labels the unit cells ( $l=0$ for the supercell), $\alpha$ labels the Cartesian coordinates, and $\hat{\boldsymbol{\kappa}}_{V C}$ is a unit vector. Explicit disorder is included in the Gamma frequencies $\omega\left(\begin{array}{c}\boldsymbol{\kappa}=\mathbf{0} \\ \nu\end{array}\right)$ and the $3 N_{a}$ components of the eigenvectors, $e\left(\begin{array}{cc}\boldsymbol{\kappa}=\mathbf{0} & b \\ \nu & \alpha\end{array}\right)$.

Physically, $S^{L, T}\left(\begin{array}{c}\boldsymbol{\kappa} \\ \omega\end{array}\right)$ represents the frequency spectrum required to create a wavepacket with a well-defined wave vector and polarization. ${ }^{17,36,66}$ For a perfect lattice, the structure factor peaks are delta functions centered at the mode frequencies, indicating that the modes are pure plane-waves (i.e., phonons). A sampling of the structure factors for the LJ argon alloys are plotted in Fig. 3 for wave vectors along the [100] and [111] directions in the $N_{0}=10$ systems. ${ }^{67}$ Well-defined peaks at all wave vectors are due to the lattice structure of the disordered systems. Typically, the structure factor for amorphous materials has well-defined peaks only for small wave vector. ${ }^{17,36}$ With increasing disorder, the structure factor spreads in width, particularly at high frequencies, which is an indication that the modes are not pure plane waves.

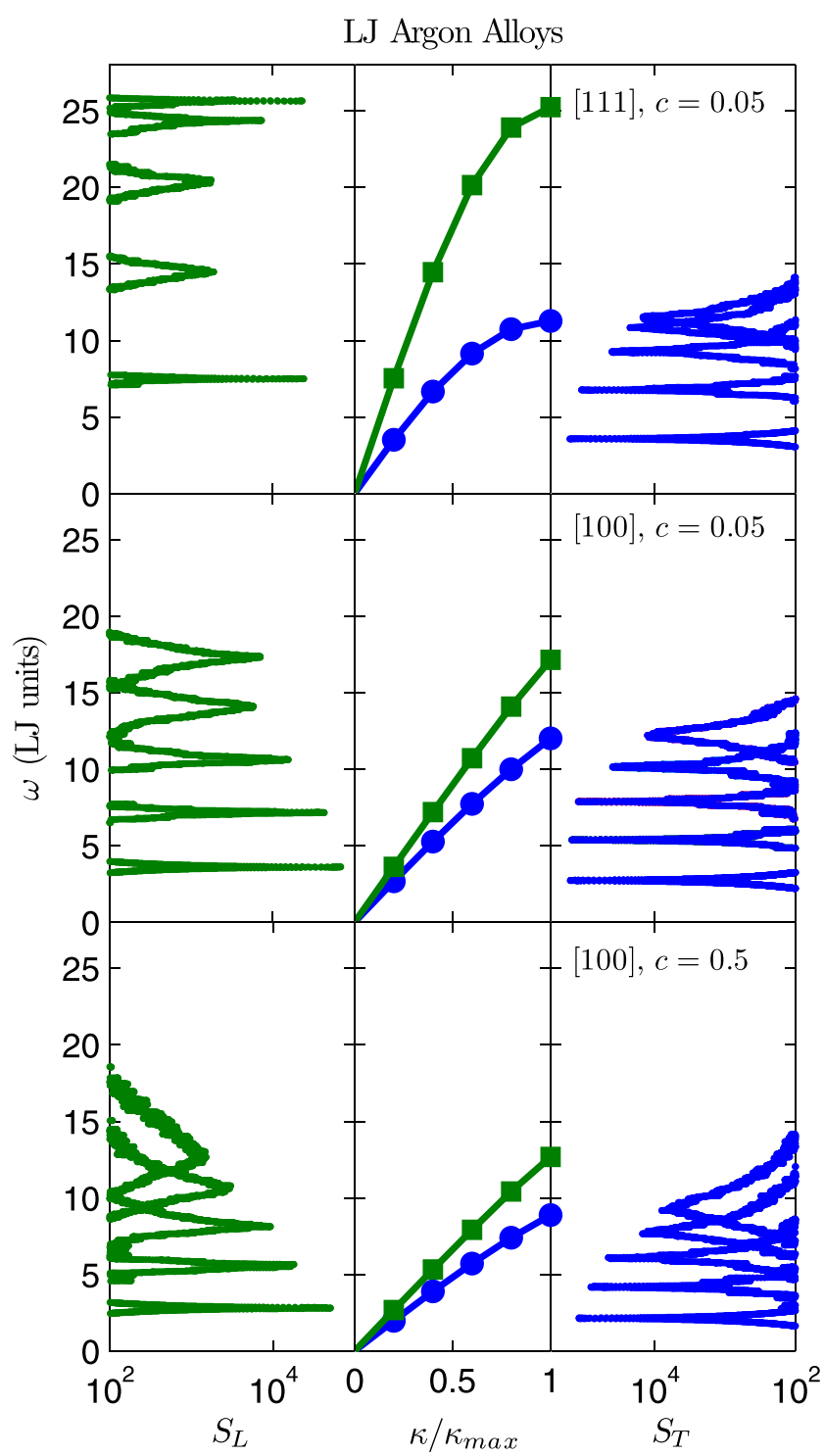

FIG. 3. Left and right panels: The structure factor for longitudinal $\left(S_{L}\right)$ and transverse $\left(S_{T}\right)$ polarizations along high-symmetry directions of the mass disordered LJ argon supercells $\left(N_{0}=10, c=0.05,0.5\right)$. Center panels: The $\mathrm{VC}$ predicted dispersion curves (solid lines) agree well with the locations of the peaks in $S_{L}$ and $S_{T}$ (data points). The wavenumber axis in the center panel is normalized by the maximum value of the wavenumber in the given direction.

From Fig. 3, an effective dispersion curve (middle panels) can be extracted by locating the peaks in the structure factors at neighboring VC wave vectors. The peaks in the structure factor are larger than the VC predicted frequencies (plotted as solid lines in Fig. 3) by at most 5\%. Similar agreement is found with the disordered SW silicon lattice supercells.

Even though there is good agreement between the VCpredicted dispersion curves and the peaks in the structure factors from Fig. 3, the effect of the width of the peaks is not clear. We will use the group velocities predicted by the VC dispersion for both LJ argon and SW silicon in the VC-NMD and VC-ALD calculations for consistency and simplicity. The validity of this group velocity choice will be discussed in Sec. III E. 


\section{Lifetimes}

\section{From VC-NMD and Gamma-NMD}

Once the group velocities are predicted using the VC dispersion, the mode lifetimes are required to predict the thermal conductivity using Eq. (1). As an alternative to the VC-ALD approach for predicting lifetimes, which is discussed in Sec. III C 2, we first use the MD simulation-based NMD method. ${ }^{33,68-70}$ In NMD, the atomic trajectories are first mapped onto the vibrational mode coordinate $q\left(\begin{array}{l}\boldsymbol{\kappa} \\ \nu\end{array} t\right)$ and its time derivative $\dot{q}\left(\begin{array}{l}\boldsymbol{\kappa} \\ \nu\end{array} ;\right)$ by $^{72}$

$q\left(\begin{array}{l}\boldsymbol{\kappa} \\ \nu\end{array} t\right)=\sum_{\alpha, b, l}^{3, n, N} \sqrt{\frac{m_{b}}{N}} u_{\alpha}\left(\begin{array}{l}l \\ b\end{array} ; t\right) e^{*}\left(\begin{array}{ll}\boldsymbol{\kappa} & b \\ \nu & \alpha\end{array}\right) \exp \left[i \boldsymbol{\kappa} \cdot \mathbf{r}_{0}\left(\begin{array}{l}l \\ 0\end{array}\right)\right]$

and

$\dot{q}\left(\begin{array}{l}\boldsymbol{\kappa} \\ \nu\end{array} t\right)=\sum_{\alpha, b, l}^{3, n, N} \sqrt{\frac{m_{b}}{N}} \dot{u}_{\alpha}\left(\begin{array}{l}l \\ b\end{array} ; t\right) e^{*}\left(\begin{array}{cc}\boldsymbol{\kappa} & b \\ \nu & \alpha\end{array}\right) \exp \left[i \boldsymbol{\kappa} \cdot \mathbf{r}_{0}\left(\begin{array}{l}l \\ 0\end{array}\right)\right]$.

Here, $m_{b}$ is the mass of the $b_{t h}$ atom in the unit cell, $u_{\alpha}$ is the $\alpha$-component of the atomic displacement from equilibrium, $\dot{u}_{\alpha}$ is the $\alpha$-component of the atomic velocity, and $t$ is time. The total energy of each vibrational mode, $E\left(\begin{array}{l}\boldsymbol{\kappa} \\ \nu\end{array} ;\right)$, is calculated from

$$
\begin{aligned}
E\left(\begin{array}{l}
\boldsymbol{\kappa} \\
\nu
\end{array} t\right)= & \frac{\omega\left(\begin{array}{l}
\boldsymbol{\kappa} \\
\nu
\end{array}\right)^{2}}{2} q\left(\begin{array}{l}
\boldsymbol{\kappa} \\
\nu
\end{array}\right)^{*} q\left(\begin{array}{l}
\boldsymbol{\kappa} \\
\nu
\end{array} t\right) \\
& +\frac{1}{2} \dot{q}\left(\begin{array}{l}
\boldsymbol{\kappa} \\
\nu
\end{array} t\right)^{*} \dot{q}\left(\begin{array}{l}
\boldsymbol{\kappa} \\
\nu
\end{array} t\right) .
\end{aligned}
$$

The vibrational mode lifetime is predicted using

$$
\tau\left(\begin{array}{l}
\boldsymbol{\kappa} \\
\nu
\end{array}\right)=\int_{0}^{t^{*}} \frac{\left\langle E\left(\begin{array}{l}
\boldsymbol{\kappa} \\
\nu
\end{array} ;\right) E\left(\begin{array}{l}
\boldsymbol{\kappa} \\
\nu
\end{array} 0\right)\right\rangle}{\left\langle E\left(\begin{array}{l}
\boldsymbol{\kappa} \\
\nu
\end{array} 0\right) E\left(\begin{array}{l}
\boldsymbol{\kappa} \\
\nu
\end{array} ; 0\right)\right\rangle} d t,
$$

where the upper integration limit $t^{*}$ is set to be much larger than the mode lifetime and the brackets indicate an ensemble average. ${ }^{70}$ The NMD calculations scale as $\left(N_{a}\right)^{2} \cdot{ }^{47}$

We perform the MD simulations using the fully disordered supercells and project onto the frequencies and eigenvectors from both the $\mathrm{VC}$ unit cell $\left[\omega\left(\begin{array}{l}\boldsymbol{\kappa} \\ \nu\end{array}\right), e\left(\begin{array}{ll}\boldsymbol{\kappa} & b \\ \nu & \alpha\end{array}\right)\right]$ and the Gamma supercell $\left[\omega\left(\begin{array}{c}\boldsymbol{\kappa}=\mathbf{0} \\ \nu\end{array}\right), e\left(\begin{array}{cc}\boldsymbol{\kappa}=\mathbf{0} & b \\ \nu & \alpha\end{array}\right)\right]$. Previous studies of disordered supercells with the NMD method have used the Gamma modes only to perform the projections. ${ }^{53,61-63,71}$ The trajectories from the MD simulations are also used in the GK method calculations (Sec. IV). The MD simulations were ten times longer than the longest lifetime in the system, which was estimated from the VC-ALD predicted lifetimes. For LJ argon and SW silicon, data were collected for $2^{20}$ and $2^{22}$ time steps and the atomic trajectories were sampled every $2^{8}$ and $2^{4}$ time steps. Ensemble averaging of the energy autocorrelations was performed using ten independent, initially randomized velocity distributions.

For the normal modes of the lattice supercell, where the energy autocorrelation follows an exponential decay, ${ }^{68,69}$ Eq. (11) is exact, but this expression becomes an approximation when using the VC normal modes to perform the mappings in Eqs. (8) and (9). Even for larger disorder $(c=0.5)$, where the energy autocorrelations deviate from an exponential decay, an effective lifetime can still be predicted using Eq. (11) (see Appendix A). The lifetimes predicted using VC-NMD and Gamma-NMD are shown in Figs. 4(a)-4(d) for the LJ argon crystal and all alloys at a temperature of $10 \mathrm{~K}$. The range of frequencies for VC-NMD and GammaNMD differ slightly due to differences in the DOS (see Fig. 2). For a small interval of frequency, there is a wider range of predicted lifetimes for Gamma-NMD. This spread is because there is no symmetry-averaging of the mode properties, which is possible for the VC by considering the crystal lattice's irreducible Brillouin zone. ${ }^{30}$

The lifetimes predicted by both VC-NMD and GammaNMD show a $\omega^{-2}$ scaling at low frequency and a $\omega^{-4}$ scaling (for the alloys) and even faster for mid-range frequencies. The $\omega^{-2}$ scaling is due to three-phonon scattering processes. $^{39,73}$ The $\omega^{-4}$ scaling is due to phonon-mass point defect scattering. ${ }^{11,37,38,40}$ A constant lifetime is observed at the highest frequencies for both VC-NMD and GammaNMD except at $c=0.5$ for VC-NMD. We are not aware of any theoretical prediction of this high-frequency behavior.

The majority of the lifetimes predicted by both VCNMD and Gamma-NMD are larger than the Ioffe-Regel (IR) $\operatorname{limit}^{74}$

$$
\tau_{I R}=\frac{2 \pi}{\omega}
$$

The physical interpretation of the IR limit is a mode that scatters in a time equal to its oscillation period. Our results suggest that the IR limit is a good lower-limit for the lifetimes predicted by VC-NMD and Gamma-NMD for LJ argon (Fig. 4) and VC-NMD for SW silicon [see Fig. 8(a) in Sec. V].

Overall, good agreement is seen in the predicted lifetimes from VC-NMD and Gamma-NMD in both magnitude and trends. The use of the VC normal modes is an approximation that becomes worse as the concentration is increased (see Appendix A), but our results suggest that the effect is only pronounced at the highest frequencies and at high alloy concentrations. The only approximation associated with Gamma-NMD is the use of the harmonic lattice dynamicspredicted frequencies and eigenvectors to map the atomic trajectories from the fully anharmonic MD simulations. This assumption has been shown to be valid for LJ argon below 
LJ Argon and Alloys, $T=10 \mathrm{~K}$

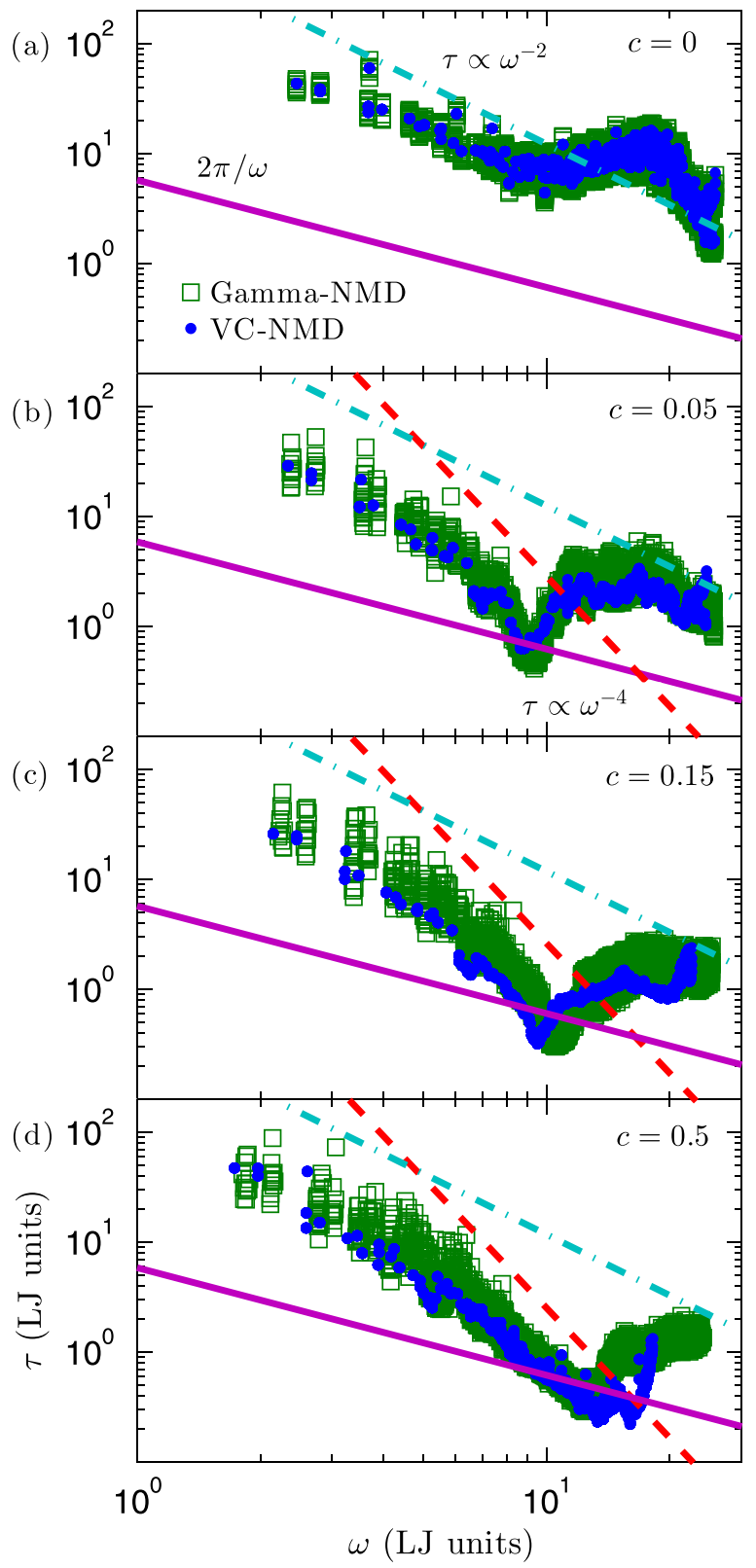

FIG. 4. Lifetimes predicted using VC-NMD and Gamma-NMD from MD simulations of (a) perfect LJ argon and (b)-(d) mass-disordered LJ alloys for $N_{0}=10 . \omega^{-2}$ and $\omega^{-4}$ scalings are observed at low to mid frequencies. For both VC-NMD and Gamma-NMD, most mode lifetimes are greater than the Ioffe-Regel limit of $2 \pi / \omega{ }^{74}$ While there is more scatter in the GammaNMD data (see Sec. III C 1), the lifetime magnitudes and trends agree well, an important consideration when comparing the VC-NMD and VC-ALD lifetimes in Fig. 5(a).

temperatures of $40 \mathrm{~K}^{69}$ Based on the good agreement with Gamma-NMD, the VC-NMD lifetimes are used along with the VC group velocities to predict thermal conductivity in Sec. IV. For Gamma-NMD, there is no accepted way to predict the mode group velocities, so that the thermal conductivity cannot be predicted using Eq. (1).

\section{From VC-ALD}

Under the VC approximation, the ALD calculations ${ }^{69}$ are performed on the conventional unit cells of LJ argon and
SW silicon with a single atomic mass based on the alloy concentration. The ALD calculations scale as $b^{4}\left(N_{0}\right)^{2} .{ }^{47}$ Disorder is not included explicitly but is treated using perturbation theory. Assuming phonon scattering mechanisms to operate independently, the effective phonon lifetime can be found using the Matthiessen rule ${ }^{15}$

$$
\frac{1}{\tau\left(\begin{array}{l}
\boldsymbol{\kappa} \\
\nu
\end{array}\right)}=\frac{1}{\tau_{p-p}\left(\begin{array}{l}
\boldsymbol{\kappa} \\
\nu
\end{array}\right)}+\frac{1}{\tau_{p-d}\left(\begin{array}{l}
\boldsymbol{\kappa} \\
\nu
\end{array}\right)},
$$

where $\tau_{p-p}\left(\begin{array}{l}\boldsymbol{\kappa} \\ \nu\end{array}\right)$ accounts for intrinsic phonon-phonon scattering and $\tau_{p-d}\left(\begin{array}{l}\boldsymbol{\kappa} \\ \nu\end{array}\right)$ accounts for phonon-defect scattering.

Phonon-phonon scattering in ALD is modeled by including three-phonon processes. ${ }^{20,22,69}$ The present study is concerned with temperatures much less than the melting temperature of either LJ argon ${ }^{51}$ or SW silicon, ${ }^{31}$ so that we believe the effects of higher-order phonon processes are negligible. ${ }^{69,75}$ We predict the phonon-phonon lifetimes using the method described in Ref. 69, with all classical expressions for the populations to remain consistent with the classical MD-based methods from Sec. III C 1.

Using perturbation theory, Tamura derived a general expression for phonon scattering by mass point defects to second order that was applied to study isotopic germanium. ${ }^{11}$ By considering the symmetry properties of the FCC lattices considered in this work, his expression reduces to

$$
\frac{1}{\tau_{p-d}\left(\begin{array}{c}
\boldsymbol{\kappa} \\
\nu
\end{array}\right)}=\frac{\pi}{2} g_{2} \omega^{2}\left(\begin{array}{l}
\boldsymbol{\kappa} \\
\nu
\end{array}\right) \operatorname{DOS}\left[\omega\left(\begin{array}{l}
\boldsymbol{\kappa} \\
\nu
\end{array}\right)\right],
$$

where

$$
g_{n}=\sum_{\mu} c^{\mu}\left(1-m^{\mu} / \bar{m}^{\mu}\right)^{n}
$$

Here, $c^{\mu}$ and $m^{\mu}$ are the concentration and mass of the $\mu$ th species and $\bar{m}^{\mu}$ is the average mass. Bond disorder can be accounted for using a similar expression with an average atomic radius or suitable scattering cross-section. ${ }^{37,38}$ For the binary LJ argon and SW silicon alloys considered here, there is one atom type in the unit cell with $\mu=i, j$, so that the alloying atom labeled by $m_{c}^{j}$ can be considered to be an "isotope" of the atom labeled $m_{1-c}^{i}$.

The lifetimes predicted by VC-ALD for LJ argon at a concentration of 0.05 are plotted in Fig. 5(a). ${ }^{76}$ Also plotted are the lifetimes for the perfect system and from the VC-NMD predictions [Fig. 4(b)] at this concentration. At low frequencies, where the DOS is Debye-like $\left[D(\omega) \propto \omega^{2}\right.$, Fig. 2], $\tau_{p-p}\left(\begin{array}{l}\boldsymbol{\kappa} \\ \nu\end{array}\right)$ scales as $\omega^{-2}$, a scaling also observed in the VC-NMD and Gamma-NMD lifetimes. Under the Debye-approximation, the phonon scattering due to mass point-defects is predicted to scale as $\omega^{-4}$ from Eq. (14). ${ }^{11,40}$ This scaling is observed in the VC-NMD, Gamma-NMD, and VC-ALD predicted lifetimes in the mid-frequency range. 


\section{LJ Argon and Alloys, $T=10 \mathrm{~K}$}

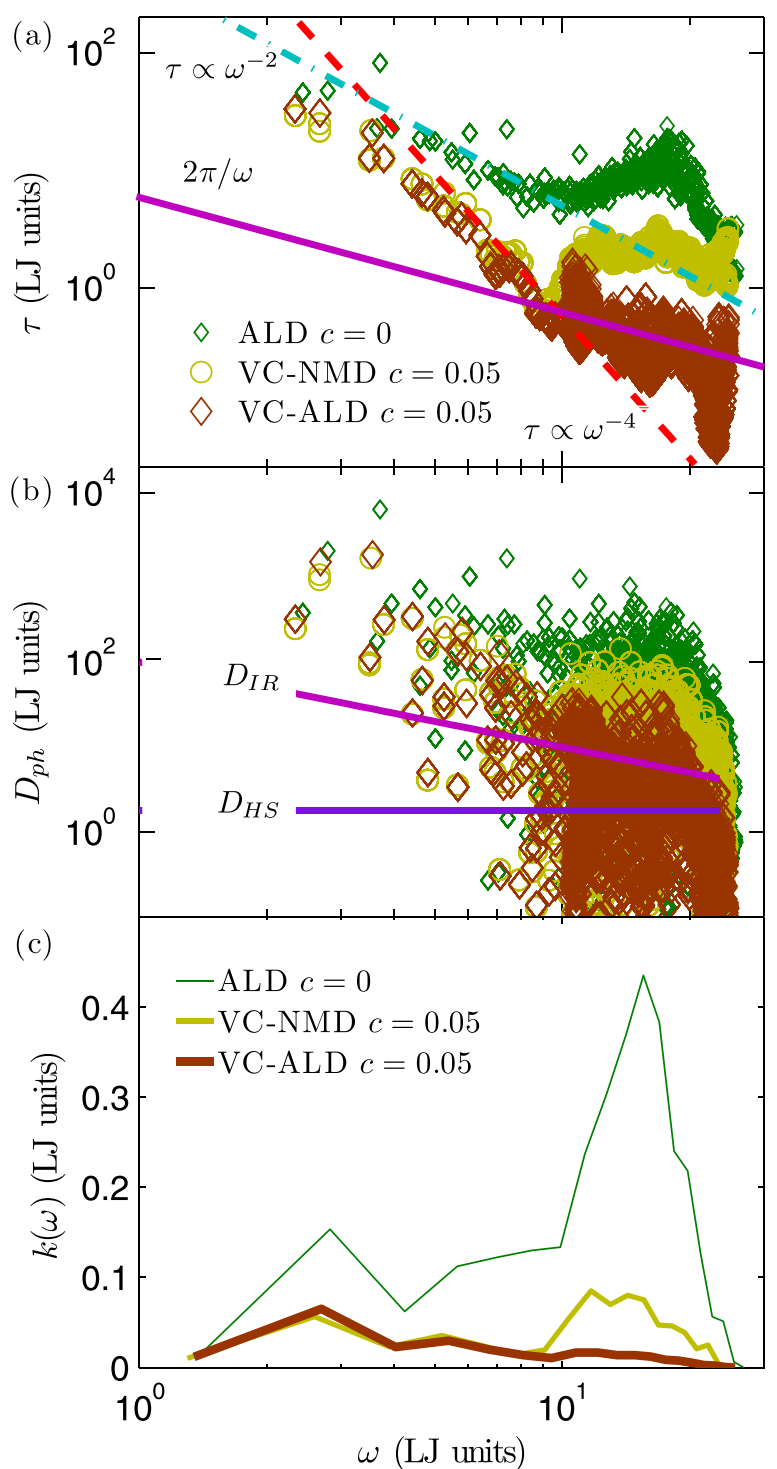

FIG. 5. (a) Predicted lifetimes using VC-NMD and VC-ALD for LJ argon ( $T=10 \mathrm{~K}, N_{0}=10$, and $c=0.05$ ). (b) Mode diffusivities compared to the high-scatter limit, $D_{H S}$ [Eq. (18)], and IR limit, $D_{I R}$ [Eq. (19)]. VC-NMD and VC-ALD predict a large number of high-frequency modes with $D_{p h}<D_{H S}$. (c) Thermal conductivity frequency spectrum, which peaks at high frequency, in contrast to SW silicon [(Fig. 8(c)].

VC-ALD does not predict the frequency-independent lifetimes at high frequency for LJ argon observed in VC-NMD and Gamma-NMD, and a significant number fall below the IR limit. The lifetimes predicted by NMD and ALD for the perfect LJ argon crystal agree within $20 \%$ on a mode-bymode basis, and the resulting thermal conductivities agree within their uncertainties (see Table I).

Tamura applied his theory to predict the reduction of lifetimes in isotopic germanium, which is weakly disordered ( $\sim 5 \%$ variation in the atomic masses). In the LJ alloys, the masses differ by a factor of three. Large mass ratios were also considered in DFT VC-ALD studies of SiGe (mass ratio of 2.6) ${ }^{20} \mathrm{PbTeSe}$ (2.6), ${ }^{22}$ and MgSiSn (4.9). ${ }^{25}$ The importance of higher-order interactions in the Tamura theory can be estimated by the disorder strength (i.e., $g_{n}$ for $n>2$ ). ${ }^{11}$ For isotopically disordered germanium, Tamura estimated
TABLE I. Thermal conductivity predictions using the VC-NMD, VC-ALD, and GK methods. For LJ argon alloys, the bulk extrapolation is used for all three methods. For SW silicon alloys, only VC-ALD and GK can be used to extrapolate a bulk thermal conductivity (see Sec. IV). For VC-NMD and GK, the uncertainties are estimated by omitting independent simulations from the ensemble averaging (see Sec. IIC). For VC-ALD, the uncertainties are estimated by omitting extrapolation points used for Eq. (21).

\begin{tabular}{lccccc}
\hline \hline$c$ & GK & VC-NMD & VC-ALD & VC-NMD* & VC-ALD* \\
\hline LJ & & & & & \\
0.00 & $3.3 \pm 0.1$ & $3.3 \pm 0.1$ & $3.4 \pm 0.1$ & & \\
0.05 & $0.80 \pm 0.07$ & $0.76 \pm 0.07$ & $0.45 \pm 0.02$ & $0.80 \pm 0.1$ & $0.52 \pm 0.05$ \\
0.15 & $0.46 \pm 0.07$ & $0.36 \pm 0.04$ & $0.24 \pm 0.01$ & $0.45 \pm 0.05$ & $0.33 \pm 0.07$ \\
0.50 & $0.38 \pm 0.07$ & $0.31 \pm 0.04$ & $0.23 \pm 0.01$ & $0.35 \pm 0.05$ & $0.31 \pm 0.07$ \\
SW & & & & & \\
0.00 & $520 \pm 30$ & & $480 \pm 20$ & & \\
0.05 & $20 \pm 2$ & & $24 \pm 2$ & & $24 \pm 2$ \\
0.15 & $9.9 \pm 0.9$ & & $12 \pm 1$ & & $12 \pm 1$ \\
0.50 & $9.3 \pm 0.9$ & & $11 \pm 1$ & & $11 \pm 1$ \\
\hline \hline
\end{tabular}

that the higher-order contributions were negligible $\left(g_{2}=5.87 \times 10^{-4}, g_{3} \sim 10^{-7}\right.$, and $\left.g_{4} \sim 10^{-7}\right) .{ }^{11}$ For LJ argon at a concentration of $0.15, g_{2}=0.3018, g_{3}=-0.3250$, and $g_{4}=0.4411$. It is possible that the neglect of the higherorder interactions in the Tamura theory is responsible for the discrepancy of the lifetimes predicted by VC-NMD and Gamma-NMD versus VC-ALD at high frequencies. Full evaluation of the higher-order interactions in the Tamura theory is of similar complexity to anharmonic phonon interaction, ${ }^{69,73,75}$ and is beyond the scope of this work.

\section{Diffusivities}

We now use the AF theory to provide a lower limit for the contribution of a given vibrational mode to thermal conductivity. While studies have been performed on alloying the amorphous phase, ${ }^{16}$ the AF theory has not been previously applied to disordered lattices. In the classical, harmonic limit for specific heat, a mode's contribution to the thermal conductivity of is determined by its diffusivity

$$
D_{p h, \mathbf{n}}\left(\begin{array}{c}
\boldsymbol{\kappa} \\
\nu
\end{array}\right)=v_{g, \mathbf{n}}^{2}\left(\begin{array}{l}
\boldsymbol{\kappa} \\
\nu
\end{array}\right) \tau\left(\begin{array}{l}
\boldsymbol{\kappa} \\
\nu
\end{array}\right)
$$

such that from Eq. (1)

$$
k_{p h, \mathbf{n}}=\sum_{\boldsymbol{\kappa}} \sum_{\nu} \frac{k_{\mathrm{B}}}{V} D_{p h, \mathbf{n}}\left(\begin{array}{l}
\boldsymbol{\kappa} \\
\nu
\end{array}\right) .
$$

The lower limit for phonon diffusivity is zero since the group velocities can be zero (e.g., optical modes at the Brillouin zone center).

In the high-scatter limit, ${ }^{2}$ the diffusivity of each mode is

$$
D_{H S}=\frac{1}{3} v_{s} a,
$$

which leads to Eq. (3). The physical interpretation of Eq. (18) is that all vibrational modes transport heat at the sound speed and have a mean free path of the lattice spacing. 
Based on the IR limit, another possible lower-bound of diffusivity is

$$
D_{I R}=\frac{2 \pi}{3} \frac{v_{s}^{2}}{\omega}
$$

To evaluate Eqs. (18) and (19), the sound speed is estimated by

$$
v_{s}=\frac{1}{3} v_{s, L}+\frac{2}{3} v_{s, T}
$$

where $v_{s, L}$ and $v_{s, T}$ are the longitudinal and transverse sound speeds calculated from the elastic constants, ${ }^{55}$ which agree within 20\% with the branch-averaged sound speeds along the high-symmetry dispersion directions [100], [110], and [111]. For LJ argon and SW silicon, $v_{s}=6.93$ (LJ units) and $5790 \mathrm{~m} / \mathrm{s}$. The Cahill-Pohl model assumes Eq. (19) for the mode diffusivities. ${ }^{2}$ As seen in Fig. 5(b) for the LJ argon alloy at a concentration of 0.05 , VC-NMD and VC-ALD predict [from Eq. (16), using the $x$-component of group velocity], a significant number of modes with $D_{p h}\left(\begin{array}{l}\boldsymbol{\kappa} \\ \nu\end{array}\right)$ less than $D_{H S}$, and $D_{I R}$ approaches $D_{H S}$ at high frequencies. For both VC-NMD and VC-ALD, we approximate $\mathbf{v}_{g, \mathbf{n}}\left(\begin{array}{l}\boldsymbol{\kappa} \\ \nu\end{array}\right)$ from the VC dispersion (Sec. III B), so that any differences in diffusivity $D_{p h}$ will come from the predicted lifetimes.

In a disordered system, modes can transport heat by harmonic coupling in the AF theory of diffusion(s). ${ }^{35}$ While the high-scatter model assumes a mode-independent diffusivity, the AF theory is capable of predicting mode-specific thermal diffusivities $D_{A F} \cdot{ }^{16,36,77}$ Since the AF theory is harmonic, the diffusivities typically diverge as the frequency approaches zero because these vibrations are long-wavelength plane waves that are weakly scattered by the disorder. ${ }^{78,79}$ The mode-specific diffusivities, $D_{A F}$, of an LJ argon amorphous phase (see Sec. IIC) ${ }^{80}$ are plotted in Fig. 6 along with $D_{H S}$ and $D_{I R}$. Except at the highest frequencies, the diffusivity of all amorphous modes can be approximated using the modeindependent diffusivity $D_{H S}$. The lower-limit $D_{I R}$ is clearly an overprediction for the amorphous mode diffusivities. Also plotted in Fig. 6 are diffusivities predicted from the AF theory for the explicitly disordered LJ argon lattice supercell alloy at a concentration of 0.5 . As expected, the AF theory predictions diverge at low frequency. ${ }^{81}$ The diffusivity of all modes are larger than $D_{H S}$ except at the highest frequencies, where they tend to zero as with the amorphous phase. This result supports the hypothesis that the lower-bound of the VC predicted phonon diffusivity should be $D_{H S}$ (and not zero as for a crystal), which is further explored in Secs. IV and V.

\section{E. Discussion}

In this section, in anticipation of the thermal conductivity predictions in Sec. IV, we discuss two possible sources of error in the VC-predicted mode properties. To start, we note that for disordered systems, it is generally only possible to assign a unique lifetime and group velocity to vibrational modes in the low-frequency, propagating limit. ${ }^{36,82}$ The

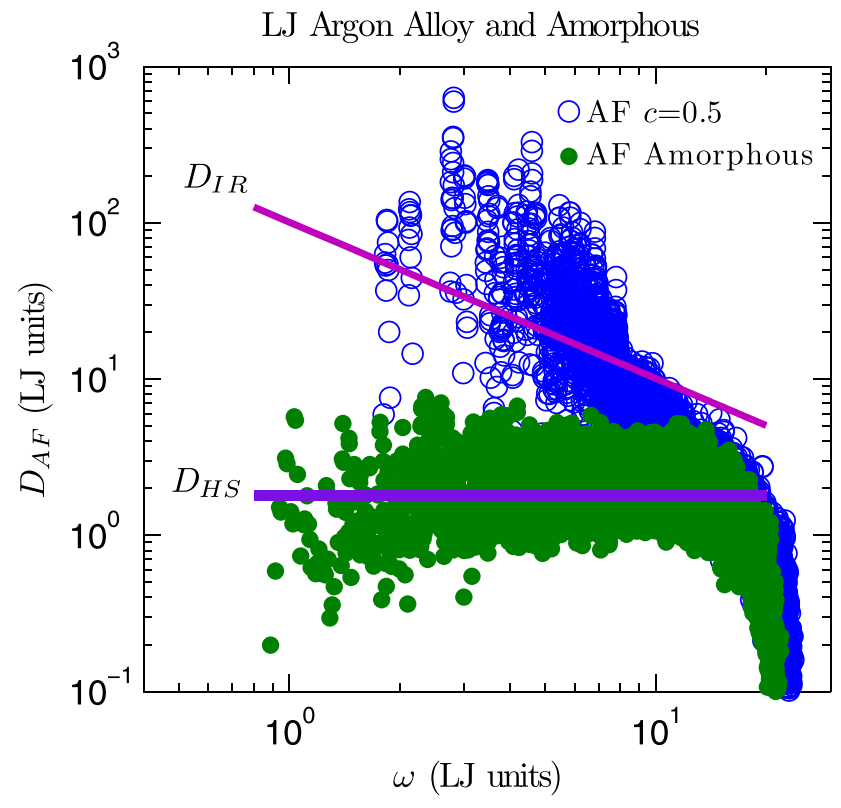

FIG. 6. AF theory predictions of disordered mode diffusivities for LJ argon alloy and amorphous phases. The amorphous phase is well-described by a mode-independent diffusivity $D_{H S}$ [Eq. (18)]. The system size for the alloy is $N_{0}=10$ (6912 atoms), and the amorphous phase has 6912 atoms. mode diffusivity is the fundamental transport
property. ${ }^{16,35,36}$

We believe that the VC-predicted group velocities, particularly for $v_{g, \mathbf{n}}\left(\begin{array}{l}\boldsymbol{\kappa} \\ \nu\end{array}\right) \approx 0$, are an underprediction of the velocity scale required to evaluate Eq. (16). This statement is supported by the AF-theory diffusivities plotted in Fig. 6, which are finite for the majority of the frequency range the LJ alloy at a concentration of 0.5 . While the diffusivity from Eq. (16) can be zero because of the VC predicted group velocities, this result is not consistent with the AF theory predictions.

The VC-NMD and Gamma-NMD predicted lifetimes are generally larger than the IR limit for LJ argon and its alloys (see Fig. 4). The constant lifetime observed at the highest frequencies for both VC-NMD (except at $c=0.5$ ) and Gamma-NMD is consistent with the plateau of mode diffusivity at high frequency predicted for a model disordered lattice, which was explained by a plateau in the vibrational mode lifetimes. ${ }^{83}$ Recently, a study of model disordered lattices predicted the mid-frequency minimum and the highfrequency plateau of the mode diffusivities. ${ }^{65}$ Similar behavior of the mode diffusivities has been observed in model jammed systems. ${ }^{79,82}$ VC-ALD predicts essentially monotonically decreasing lifetimes with increasing frequency for the LJ argon alloys [Fig. 5(a)] with many falling below the IR limit. Because VC-NMD and VC-ALD use the same values for $v_{g, \mathbf{n}}\left(\begin{array}{l}\boldsymbol{\kappa} \\ \nu\end{array}\right)$, the mode diffusivities will therefore be underpredicted for VC-ALD compared to VC-NMD for the $\mathrm{LJ}$ argon alloys because of the lifetime underprediction.

\section{THERMAL CONDUCTIVITY PREDICTION}

The thermal conductivities of the LJ systems can now be predicted from Eq. (1) using the vibrational mode 
properties from VC-NMD and VC-ALD. Given the discussion regarding the VC-predicted mode properties in Sec. IIIE, we also predict thermal conductivity using the equilibrium MD-based GK method, which is a top-down method that does not make any approximations about the nature of the vibrational modes. Thermal conductivities predicted by the GK method naturally capture all scattering mechanisms. ${ }^{53,60,84}$ The heat current was computed every ten time steps from the same atomic trajectories (positions and velocities) used for the VC-NMD and Gamma-NMD calculations. The thermal conductivity is determined from the maximum of the integral of the heat current autocorrelation function.

The thermal conductivities predicted by VC-NMD, VC$\mathrm{ALD}$, and GK are system size-dependent [i.e., $k=k\left(N_{0}\right)$ ] for all lattices and methods except perfect LJ argon from GK. ${ }^{33}$ To predict a bulk thermal conductivity, $k_{b u l k}$, a linear extrapolation procedure is used, whereby

$$
\frac{k\left(N_{0}\right)}{k_{\text {bulk }}}=1-\frac{c_{0}}{N_{0}},
$$

where $c_{0}$ is a constant. ${ }^{23,24,47}$ The thermal conductivity is predicted for varying system sizes and the bulk thermal conductivity is obtained by fitting Eq. (21) to the data. For VCNMD and VC-ALD, the validity of Eq. (21) requires that the low-frequency modes be dominated by phonon-phonon scattering (i.e., $\tau \propto \omega^{-2}$ ) and follow the Debye approximation with respect to the group velocity and DOS. ${ }^{23,24}$ For the LJ argon alloys, this requirement is satisfied for modest system sizes (for $N_{0}=6$ to 12), so that both VC-NMD and VC-ALD thermal conductivity predictions can be extrapolated to a bulk value.

Bulk thermal conductivity predictions for the LJ argon alloys using VC-NMD, VC-ALD, and GK are tabulated in Table I and plotted in Fig. 7. Also plotted in Fig. 7 is the high-scatter thermal conductivity prediction $k_{H S}$ [Eq. (3)]. The thermal conductivity predicted for the LJ amorphous phase by GK is $0.17 \mathrm{~W} / \mathrm{m}-\mathrm{K}$, which is in good agreement with $k_{H S}(0.16 \mathrm{~W} / \mathrm{m}-\mathrm{K})$ for the perfect crystal. The predicted thermal conductivities of the LJ argon alloys at high concentration are a factor of two to three larger than $k_{H S}$. While agreement between the three methods is found for the perfect crystal, VC-NMD and VC-ALD underpredict the alloy thermal conductivities compared to GK. The underprediction is modest for VC-NMD, where $k_{N M D}$ is $80 \%$ of $k_{G K}$ or greater for all concentrations. The VC-ALD method significantly underpredicts the thermal conductivity of the LJ argon alloys. The largest deviation is at a concentration of 0.05 , where $k_{V C-A L D}$ is $56 \%$ of $k_{G K}$.

In Sec. III D, we argued for the existence of a minimum mode diffusivity, $D_{H S}$ [Eq. (18)]. As shown in Fig. 5(b), the diffusivities of many high-frequency modes in the $\mathrm{LJ}$ alloys, predicted by both VC-NMD and VC-ALD, fall below this limit. Based on this observation, we propose that any diffusivity below the limit be set to $D_{H S}$ for thermal conductivity prediction. The results of this adjustment, referred to as VCNMD* and VC-ALD*, are plotted in Fig. 7 and included in Table I. The adjusted thermal conductivities predicted by

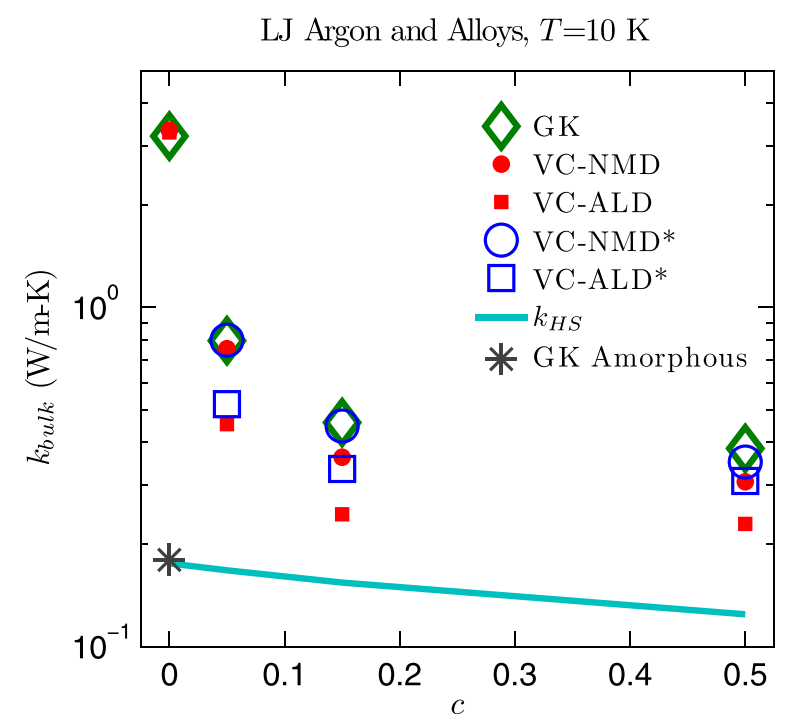

FIG. 7. Thermal conductivity predictions for LJ argon and alloys at $T=10 \mathrm{~K}$ using the VC-NMD, VC-ALD, and GK methods. The high-scatter thermal conductivity prediction $k_{H S}$ [Eq. (3)] and the high-scatter adjusted VC-NMD* and VC-ALD* are also plotted.

VC-NMD ${ }^{*}$ are now within $10 \%$ of the GK value for all concentrations, which is within the prediction uncertainties. Combined with $D_{H S}$, we believe that the VC-NMD predicted diffusivities are good representations for the explicitly disordered modes present in the MD simulations. Another possible adjustment, $D_{I R}$ [Eq. (19)], results in a thermal conductivity of $0.94 \pm 0.09 \mathrm{~W} / \mathrm{m}-\mathrm{K}$ for the LJ argon alloy at a concentration of 0.05 , well above the value predicted by GK. We also note that the thermal conductivity of the amorphous phase is well-modeled by a mode-independent diffusivity $D_{H S}$, while $D_{I R}$ overpredicts for all modes in the amorphous phase (see Fig. 6). Thus, we believe that $D_{H S}$ is the more appropriate high-scatter limit.

By applying the high-scatter limit adjustment VC-ALD*, the thermal conductivities are brought into marginally better agreement with the GK values, worst for a concentration of 0.05 , where $k_{V C-A L D^{*}}$ is $65 \%$ of $k_{G K}$. As seen in Fig. $5(\mathrm{~b})$, the VC-ALD method fails to accurately predict the highfrequency mode diffusivities for LJ argon alloys. Since the group velocities are the same for VC-NMD and VC-ALD, the underprediction of the high-frequency diffusivities is due to the underprediction of the high-frequency mode lifetimes from VC-ALD compared to VC-NMD. We know that the VC-NMD predicted lifetimes are more accurate values due to their agreement with Gamma-NMD [Fig. 4].

The thermal conductivity spectrum, defined as the contribution to thermal conductivity of modes at a given frequency, is plotted in Fig. 5(c) for VC-NMD and VC-ALD for the perfect crystal and the alloy with a concentration of 0.05 . The thermal conductivity of LJ argon and its alloys has important contributions from high-frequency modes. VC-ALD underpredicts the high-frequency diffusivities compared to VC-NMD, which leads to an underprediction of the high-frequency thermal conductivity spectrum compared to VC-NMD. This result can be traced back to an underprediction of the high-frequency lifetimes compared to VC-NMD and Gamma-NMD [Fig. 5(a)]. 


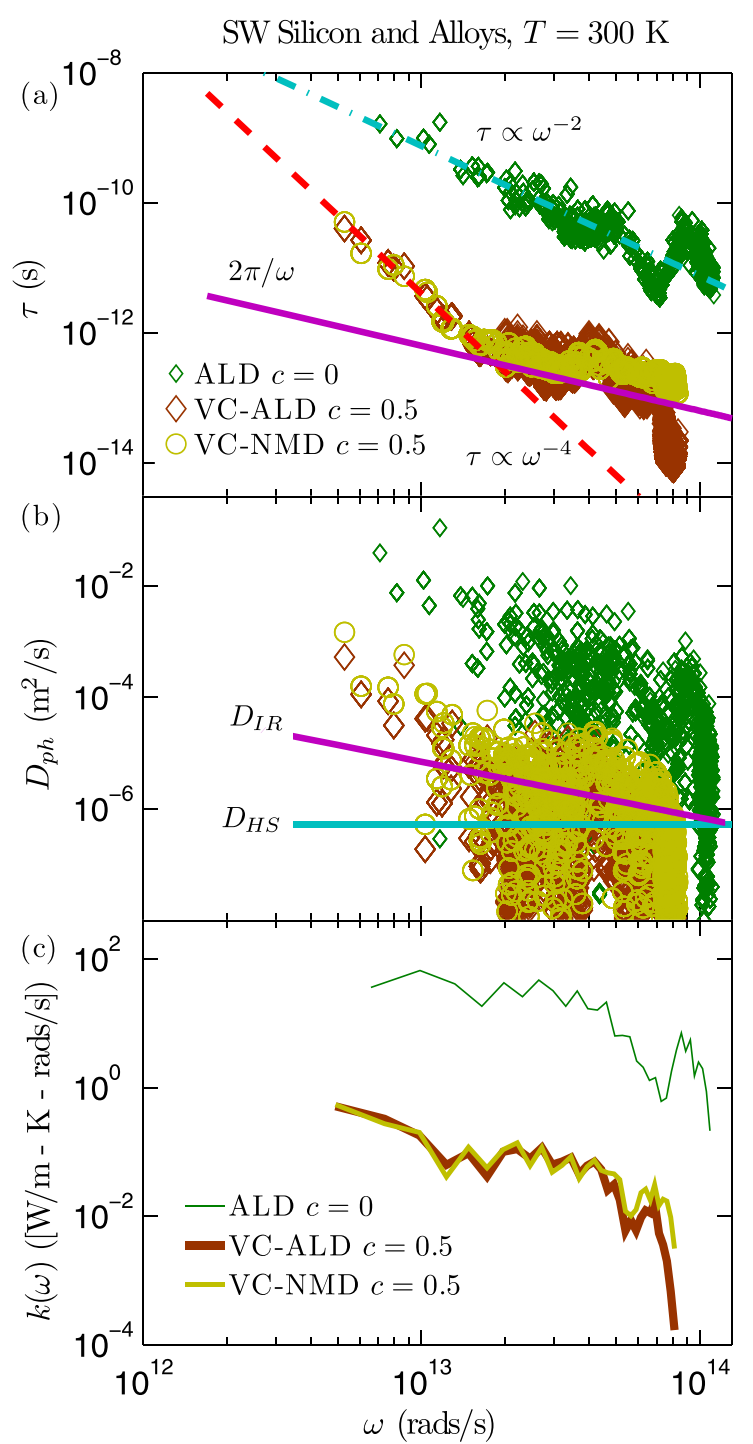

FIG. 8. (a) Predicted lifetimes using VC-NMD and VC-ALD for SW silicon ( $T=300 \mathrm{~K}, N_{0}=8$, and $c=0.05$ ). (b) Mode diffusivities compared to the high-scatter limit, $D_{H S}$ [Eq. (18)], and the IR limit, $D_{I R}$ [Eq. (19)]. VC-NMD and VC-ALD predict a large number of high-frequency modes with $D_{p h}<D_{H S}$, as seen in the LJ argon alloys [Fig. 5(b)]. (c) Thermal conductivity frequency spectra, which peak at low frequency, in contrast to LJ argon [Fig. 5(c)].

\section{SW SILICON}

The failure of VC-ALD to predict the thermal conductivities of the LJ alloys is due to an underprediction of the high-frequency mode lifetimes, which make an important contribution to the thermal conductivity [see Secs. IIID and IV, Figs. 5(a) and 5(c)]. To provide a contrast, we now predict the vibrational mode properties and thermal conductivity for bulk and alloyed SW silicon, where it is known that low-frequency modes dominate the thermal conductivity. ${ }^{54,85}$ The lifetimes for the perfect crystal and an alloy with a concentration of 0.5 predicted by VC-NMD and VC-ALD are plotted in Fig. 8(a). The VCNMD predicted lifetimes are generally larger than the IR limit for SW silicon alloys, similar to the VC-NMD predictions for the LJ argon alloys (Fig. 4). Unlike the LJ argon alloys, the VC-NMD and VC-ALD predicted lifetimes agree over most of the frequency spectrum, except at the highest frequencies, where VC-ALD underpredicts VC-NMD and falls below the IR limit. The highfrequency plateau of the VC-NMD predicted lifetimes for LJ argon (Fig. 4) is not seen for SW silicon. As seen in Figs. 5(b) and 8(b), VC-NMD and VC-ALD both predict a significant number of modes with $D_{p h}\left(\begin{array}{l}\boldsymbol{\kappa} \\ \nu\end{array}\right)$ less than $D_{H S}$ for both the LJ argon and SW silicon alloys.

The thermal conductivity spectra for bulk SW silicon and an alloy with a concentration of 0.5 are plotted in Fig. 8(c). For bulk and the alloy, the thermal conductivity is dominated by low-frequency modes, so that large systemsizes are needed to satisfy the extrapolation requirements and only GK and VC-ALD can be used to predict a bulk value from Eq. (21). While a previous study found that it was necessary to use cell sizes of $N_{0}=60$ for Tersoff silicon alloys ${ }^{71}$ we find that Eq. (21) is valid for SW silicon and $38 \leq N_{0} \leq 42$. This system-size requirement highlights the efficiency of the VC-ALD method compared to VC-NMD, which is necessary when computationally expensive DFT calculations are used. ${ }^{12,20,22,24,86,87}$ The bulk thermal conductivity predictions for VC-ALD and GK are shown in Table I and plotted in Fig. 9. The alloy thermal conductivities predicted by VC-ALD are $20 \%$ larger than those from $\mathrm{GK}$, in contrast to VC-ALD underpredicting for LJ argon alloys. This overprediction by VC-ALD compared to GK is close to the overprediction (15\%) of VC-ALD using DFT calculations of $\mathrm{SiGe}$ alloys compared to experiment without including disorder explicitly. ${ }^{20}$

The predicted thermal conductivities for the SW silicon alloys at all concentrations are over an order of magnitude larger than the high-scatter prediction, $k_{H S}$. Because the thermal transport in SW silicon is dominated by

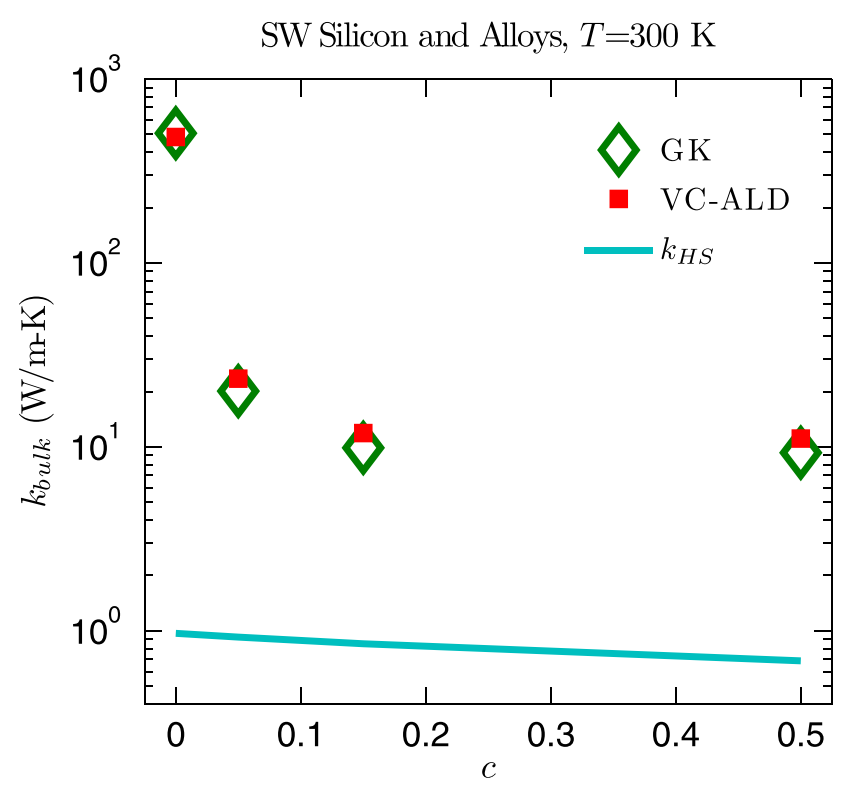

FIG. 9. Thermal conductivity predictions for SW silicon and alloys at a temperature of $300 \mathrm{~K}$ using the VC-ALD and GK methods. The highscatter thermal conductivity prediction $k_{H S}$ is also plotted. The adjusted VC-ALD* is not shown since it differs by less than one percent compared to VC-ALD. 
low-frequency modes, the high-scatter adjustment VC$\mathrm{ALD}^{*}$ is within one percent compared to the unadjusted VC-ALD. While higher-order interactions in the Tamura theory may be responsible for the discrepancy of the lifetimes predicted by VC-NMD and VC-ALD in SW silicon at the highest frequencies [Fig. 8(a)], this effect is not important to the overall thermal transport. VC-ALD predicts accurate alloy thermal conductivities for SW silicon because it is a low-frequency dominated material, which is the frequency range where the standard application of the Tamura theory is valid. ${ }^{11}$

\section{SUMMARY}

In this study, we investigated the use of the VC approximation for predicting the vibrational mode properties and thermal conductivity of LJ argon and SW silicon alloys by a detailed comparison of the VC-NMD, VC-ALD, and GK methods. By using computationally inexpensive empirical potentials, we self-consistently studied the effects of disorder both explicitly (Secs. III A, III B, IIIC I, III D, and V) and as a perturbation (Secs. III C and V). By spanning a range of disorder, the limits of the perturbative models were examined. A breakdown of the VC-ALD method was identified for LJ argon alloys by a comparison with the VC-NMD method in Sec. IIIC and a correction was suggested in Sec. IV. The mode properties and thermal conductivity of the SW silicon alloys were predicted in Sec. V and provided a contrast to the LJ argon alloys, which have a different thermal conductivity spectrum.

The results for the SW silicon and LJ argon alloys suggest that modeling of thermal transport in ordered and disordered lattices can be separated into two broad groups: low-frequency dominated and full-spectrum materials. Materials dominated by low-frequency modes tend to have high thermal conductivities that are significantly larger than the high-scatter limit [Eq. (3)], which is due to the large group velocities and long lifetimes of lowfrequency modes. ${ }^{12-14,20,41-43,88}$ These low-frequency modes closely follow the scalings predicted by the perturbative VC-ALD models, which are valid at lowfrequencies.

$\mathrm{LJ}$ argon is a material whose thermal transport has significant contribution from high-frequency modes, even for the bulk [see Fig. 5(c)]. This high-frequency range is where we predict that the perturbative Tamura theory will have non-negligible contributions from higher-order interactions (see Sec. IIIC). While the higher-order interactions in the Tamura theory are also predicted to be non-negligible for SW silicon, this does not affect the thermal conductivity predictions significantly because high-frequency modes are not important to thermal transport. The negligible contributions of high-frequency modes is demonstrated by experimental measurements of the thermal conductivity of SiGe alloys, which exceed the high-scatter limit by more than an order of magnitude at room temperature for all compositions. ${ }^{2,42,43,88}$ Experimentally accurate theoretical predictions ${ }^{20}$ also demonstrate that high-frequency modes are unimportant to thermal transport, although they do serve as important scattering channels. ${ }^{19}$

The VC-ALD method provides a computationally inexpensive framework, which is essential when using $a b$ initio methods for predicting thermal conductivity. ${ }^{12,19-26}$ Based on our results, we believe that the Tamura theory breaks down for mode diffusivities predicted to be below the high-scatter limit, $D_{H S}$ [Eq. (18)]. This breakdown may be true for the high-frequency modes of any disordered lattice $^{83}$ and the high-scatter limit $D_{H S}$ should be considered whenever the perturbative VC-ALD method is used. Although the high-scatter limit of diffusivity is usually interpreted as a minimum mean free path, ${ }^{1,2,83,89}$ we find that this concept is not necessary for interpreting the results of this work. In a disordered lattice, the fundamental quantities are the mode lifetime and diffusivity ${ }^{17,35,65,74,79,82,83}$ and the VC predicted group velocity is an approximation.

\section{ACKNOWLEDGMENTS}

This work was supported by AFOSR Award FA95501010098 and by a grant of computer time from the DOD High Performance Computing Modernization Program at the US Army Engineer Research and Development Center. We thank Davide Donadio, Jivtesh Garg, Asad Hasan, Ankit Jain, Craig Maloney, and Zhiting Tian for their helpful discussions.

\section{APPENDIX A: NMD USING NON-EXACT NORMAL MODES}

For a normal mode of the lattice supercell used for the MD simulations (i.e., a Gamma mode), the total energy autocorrelation is an exponential function with a decay time $\tau\left(\begin{array}{l}\boldsymbol{\kappa} \\ \nu\end{array}\right)$ and the kinetic energy autocorrelation is a exponentially damped sinusoidal oscillation with frequency $2 \omega\left(\begin{array}{l}\boldsymbol{\kappa} \\ \nu\end{array}\right) \cdot{ }^{90}$ When projecting MD simulations of the explicitly disordered lattice supercells onto the VC normal modes, the energy autocorrelation functions do not always follow these simple functional forms, as shown in Fig. 10 for two modes in the LJ alloy at a concentration of 0.5 . By calculating the mode kinetic energy in the frequency-domain, $\Phi,{ }^{70}$ artifacts such as multiple peaks are observed (see main plot).

These artifacts are not surprising given two considerations: (i) the MD simulations contain explicit disorder that influences the atomic trajectories, and (ii) the VC-normal modes are not the exact normal modes of the explicitly disordered lattice supercells. An effective lifetime can be predicted using Eq. (11) because the VC total mode energy autocorrelations still decay to zero in a finite time. This result is to be expected given that the atomic trajectories contain information about the lattice energy, which from general statistical physics principles will have exponential relaxation behavior in an equilibrium ensemble. $^{91-93}$ 


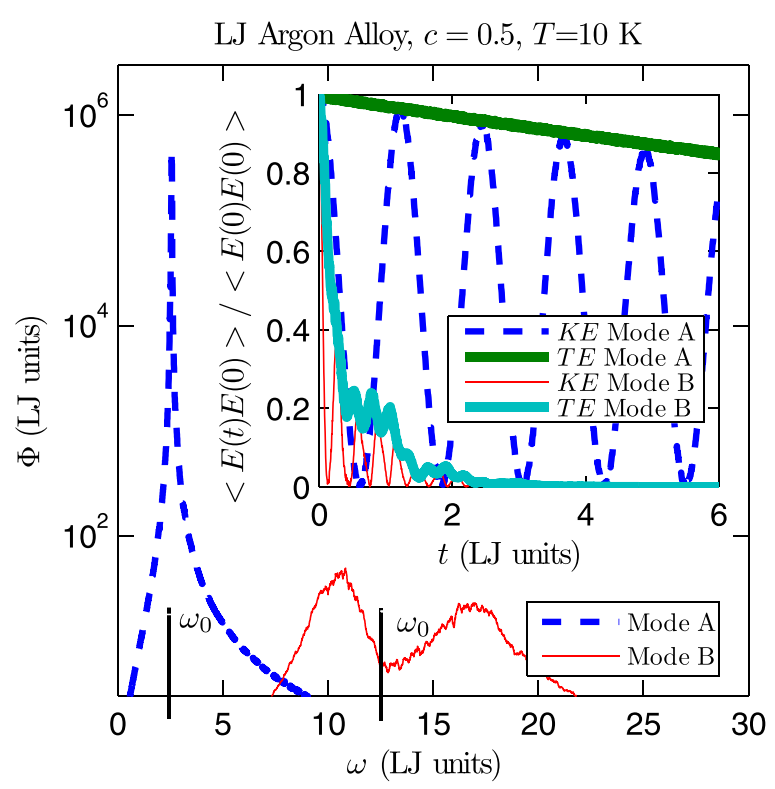

FIG. 10. The normal mode kinetic energy, $\Phi$, of two modes (A and B) at wavevector $\left[\begin{array}{lll}0.25 & 0 & 0\end{array}\right]$ calculated using VC-NMD for a mass disordered LJ FCC supercell $\left(N_{0}=8\right.$ and $\left.c=0.5\right)$ is shown in the main figure. The VC dispersion-predicted peaks are labeled by $\omega_{0}$. The inset shows the same mode's energy [kinetic $(K E)$ and total $(T E)$ ] autocorrelation functions. Note the additional oscillation effects in the KE and TE autocorrelation functions for Mode B, which are due to the two peaks in $\Phi$. A mode lifetime can be extracted unambiguously using the integral of the TE autocorrelation function [Eq. (11) in Sec. III C 1].

${ }^{1}$ J. E. Graebner, B. Golding, and L. C. Allen, Phys. Rev. B 34, 5696-5701 (1986).

${ }^{2}$ D. Cahill and R. Pohl, Ann. Rev. Phys. Chem. 39, 93-121 (1988).

${ }^{3}$ X. Lu, M. C. Arduini-Schuster, J. Kuhn, O. Nilsson, J. Fricke, and R. W. Pekala, Science 255, 971 (1992).

${ }^{4}$ G. Chen, M. S. Dresselhaus, G. Dresselhaus, J.-P. Fleurial, and T. Caillat, Int. Mater. Rev. 48, 45-66 (2003).

${ }^{5}$ D. R. Clarke and S. R. Phillpot, Mater. Today 8, 22-29 (2005).

${ }^{6}$ G. J. Snyder and E. S. Toberer, Nature Mater. 7, 105 (2008).

${ }^{7}$ A. J. Minnich, M. S. Dresselhaus, F. Ren, and G. Chen, Energy Environ. Sci. 2, 466-479 (2009).

${ }^{8}$ E. S. Toberer, A. Zevalkink, and G. J. Snyder, J. Mater. Chem. 21, 15843-15852 (2011).

${ }^{9}$ M. Zebarjadi, K. Esfarjani, M. S. Dresselhaus, Z. F. Ren, and G. Chen, Energy Environ. Sci. 5, 5147 (2012).

${ }^{10}$ S. N. Schiffres, K. H. Kim, L. Hu, A. J. H. McGaughey, M. F. Islam, and J. A. Malen, Adv. Funct. Mater. 22, 5251 (2012).

${ }^{11}$ S.-i. Tamura, Phys. Rev. B 27, 858-866 (1983).

${ }^{12}$ L. Lindsay, D. A. Broido, and T. L. Reinecke, Phys. Rev. Lett. 109, 095901 (2012).

${ }^{13}$ B. Abeles, D. S. Beers, G. D. Cody, and J. P. Dismukes, Phys. Rev. 125, 44-46 (1962).

${ }^{14}$ B. Abeles, Phys. Rev. 131, 1906-1911 (1963).

${ }^{15}$ J. M. Ziman, Electrons and Phonons (Oxford, New York, 2001).

${ }^{16}$ J. L. Feldman, M. D. Kluge, P. B. Allen, and F. Wooten, Phys. Rev. B 48, 12589-12602 (1993).

${ }^{17}$ P. B. Allen, J. L. Feldman, J. Fabian, and F. Wooten, Philos. Mag. B 79, 1715 (1999).

${ }^{18}$ D. A. Broido, M. Maloney, G. Birner, N. Mingo, and D. Stewart, Appl. Phys. Lett. 91, 231922 (2007).

${ }^{19}$ A. Ward and D. A. Broido, Phys. Rev. B 81, 085205 (2010).

${ }^{20}$ J. Garg, N. Bonini, B. Kozinsky, and N. Marzari, Phys. Rev. Lett. 106, 045901 (2011).

${ }^{21}$ T. Shiga, J. Shiomi, J. Ma, O. Delaire, T. Radzynski, A. Lusakowski, K. Esfarjani, and G. Chen, Phys. Rev. B 85, 155203 (2012).

${ }^{22}$ Z. Tian, J. Garg, K. Esfarjani, T. Shiga, J. Shiomi, and G. Chen, Phys. Rev. B 85, 184303 (2012).

${ }^{23}$ J. Shiomi, K. Esfarjani, and G. Chen, Phys. Rev. B 84, 104302 (2011).

${ }^{24}$ K. Esfarjani, G. Chen, and H. T. Stokes, Phys. Rev. B 84, 085204 (2011).
${ }^{25}$ W. Li, L. Lindsay, D. A. Broido, D. A. Stewart, and N. Mingo, Phys. Rev. B 86, 174307 (2012)

${ }^{26}$ M. N. Luckyanova, J. Garg, K. Esfarjani, A. Jandl, M. T. Bulsara, A. J. Schmidt, A. J. Minnich, S. Chen, M. S. Dresselhaus, Z. Ren et al., Science 338, 936 (2012)

${ }^{27}$ A. Ward, D. A. Broido, D. A. Stewart, and G. Deinzer, Phys. Rev. B 80, 125203 (2009).

${ }^{28}$ H. Bao, B. Qiu, Y. Zhang, and X. Ruan, J. Quantitative Spectrosc. Radiat. Transfer 113, 1683 (2012).

${ }^{29}$ G. C. Sosso, D. Donadio, S. Caravati, J. Behler, and M. Bernasconi, Phys. Rev. B 86, 104301 (2012)

${ }^{30}$ N. W. Ashcroft and N. D. Mermin, Solid State Physics (Saunders, Fort Worth, 1976).

${ }^{31}$ F. H. Stillinger and T. A. Weber, Phys. Rev. B 31, 5262-5271 (1985).

${ }^{32}$ D. A. McQuarrie, Statistical Mechanics (University Science Books, Sausalito, 2000).

${ }^{33}$ A. J. H. McGaughey and M. Kaviany, Phys. Rev. B 69, 094303 (2004).

${ }^{34}$ J. V. Goicochea, M. Madrid, and C. H. Amon, J. Heat Transfer 132, 012401 (2010).

${ }^{35}$ P. B. Allen and J. L. Feldman, Phys. Rev. B 48, 12581-12588 (1993).

${ }^{36}$ J. L. Feldman, P. B. Allen, and S. R. Bickham, Phys. Rev. B 59, 3551-3559 (1999) .

${ }^{37}$ P. G. Klemens, Proc. Phys. Soc. Sec. A 68, 1113-1128 (1955).

${ }^{38}$ P. G. Klemens, Proc. Phys. Soc. Sec. A 70, 833 (1957).

${ }^{39}$ J. Callaway, Phys. Rev. 113, 1046 (1959).

${ }^{40}$ D. C. Mattis, Phys. Rev. 107, 1736-1736 (1957).

${ }^{41}$ W. A. Kamitakahara and B. N. Brockhouse, Phys. Rev. B 10, 1200-1212 (1974).

${ }^{42}$ D. G. Cahill and F. Watanabe, Phys. Rev. B 70, 235322 (2004).

${ }^{43}$ D. G. Cahill, F. Watanabe, A. Rockett, and C. B. Vining, Phys. Rev. B 71, 235202 (2005).

${ }^{44}$ A. Skye and P. K. Schelling, J. Appl. Phys. 103, 113524 (2008).

${ }^{45}$ E. S. Landry and A. J. H. McGaughey, Phys. Rev. B 80, 165304 (2009).

${ }^{46}$ Z. Tian, K. Esfarjani, and G. Chen, Phys. Rev. B 86, 235304 (2012).

${ }^{47}$ J. E. Turney, Ph.D. disertation, Carnegie Mellon University, Pittsburgh, PA, 2009.

${ }^{48}$ I. Kudman, J. Mater. Sci. 7, 1027 (1972).

${ }^{49}$ Y. Pei, X. Shi, A. LaLonde, H. Wang, L. Chen, and G. J. Snyder, Nature 473, 66 (2011).

${ }^{50}$ K. Momma and F. Izumi, J. Appl. Crystallogr. 41, 653-658 (2008).

${ }^{51}$ A. J. H. McGaughey, Ph.D. disertation, University of Michigan, Ann Arbor, MI, 2004.

${ }^{52}$ S. Plimpton, J. Comput. Phys. 117, 1 (1995).

${ }^{53}$ Y. He, I. Savic, D. Donadio, and G. Galli, Phys. Chem. Chem. Phys. 14, 16209-16222 (2012).

${ }^{54}$ D. P. Sellan, J. E. Turney, A. J. H. McGaughey, and C. H. Amon, J. Appl. Phys. 108, 113524 (2010).

${ }^{55}$ J. D. Gale and A. L. Rohl, Mol. Simul. 29, 291 (2003).

${ }^{56}$ A. M. Bouchard, R. Biswas, W. A. Kamitakahara, G. S. Grest, and C. M. Soukoulis, Phys. Rev. B 38, 10499-10506 (1988).

${ }^{57}$ J. C. Duda, T. S. English, D. A. Jordan, P. M. Norris, and W. A. Soffa, J. Phys.: Condensed Matter 23, 205401 (2011).

${ }^{58}$ Q.-J. Chu and Z.-Q. Zhang, Phys. Rev. B 39, 7120-7131 (1989).

${ }^{59}$ A. J. H. McGaughey and M. Kaviany, in Advances in Heat Transfer, edited by G. A. Greene, Y. I. Cho, J. P. Hartnett, and A. Bar-Cohen (Elsevier, 2006), Vol. 39, pp. 169-255.

${ }^{60}$ Y. He, D. Donadio, J.-H. Lee, J. C. Grossman, and G. Galli, ACS Nano 5, 1839-1844 (2011).

${ }^{61}$ Y. He, D. Donadio, and G. Galli, Appl. Phys. Lett. 98, 144101 (2011).

${ }^{62}$ D. Donadio and G. Galli, Phys. Rev. Lett. 102, 195901 (2009).

${ }^{63}$ T. Hori, T. Shiga, and J. Shiomi, J. Appl. Phys. 113, 203514 (2013).

${ }^{64}$ S. Volz and G. Chen, Phys. Rev. B 61, 2651-2656 (2000).

${ }^{65}$ Y. M. Beltukov, V. I. Kozub, and D. A. Parshin, Phys. Rev. B 87, 134203 (2013).

${ }^{66}$ N. L. Green, D. Kaya, C. E. Maloney, and M. F. Islam, Phys. Rev. E 83, 051404 (2011).

${ }^{67}$ Due to the finite-size system, the delta function in Eq. (5) is broadened using a Lorentzian function with a full-width at half maximum set to $20 \delta_{\omega, \text { avg }}$, where $\delta_{\omega, \text { avg }}$ is the average frequency spacing (Ref. 16).

${ }^{68}$ A. J. C. Ladd, B. Moran, and W. G. Hoover, Phys. Rev. B 34, 5058-5064 (1986).

${ }^{69}$ J. E. Turney, E. S. Landry, A. J. H. McGaughey, and C. H. Amon, Phys. Rev. B 79, 064301 (2009). 
${ }^{70}$ J. M. Larkin, J. E. Turney, A. D. Massicotte, C. H. Amon, and A. J. H. McGaughey, "Comparison and evaluation of spectral energy methods for predicting phone properties," J. Comput. Theor. Nanosci. (to be published).

${ }^{71}$ Y. He, D. Donadio, and G. Galli, Nano Lett. 11, 3608-3611 (2011).

${ }^{72}$ M. T. Dove, Introduction to Lattice Dynamics (Cambridge, Cambridge, 1993).

${ }^{73}$ A. A. Maradudin and A. E. Fein, Phys. Rev. 128, 2589-2608 (1962).

${ }^{74}$ S. N. Taraskin and S. R. Elliott, Philos. Mag. Part B 79, 1747-1754 (1999).

${ }^{75}$ D. J. Ecsedy and P. G. Klemens, Phys. Rev. B 15, 5957-5962 (1977).

${ }^{76}$ To perform the calculation of Eq. (14), it is necessary to broaden the DOS using a Lorentzian function (Ref. 11). We use a value of $100 \delta_{\omega, \text { avg }}$.

${ }^{77}$ S. Shenogin, A. Bodapati, P. Keblinski, and A. J. H. McGaughey, J. Appl. Phys. 105, 034906 (2009).

${ }^{78} \mathrm{P}$. Sheng, Introduction to Wave Scattering: Localization and Mesoscopic Phenomena (Springer, 2006).

${ }^{79}$ V. Vitelli, N. Xu, M. Wyart, A. J. Liu, and S. R. Nagel, Phys. Rev. E 81, 021301 (2010)

${ }^{80}$ For a finite system, the AF theory requires a frequency broadening to predict the mode-specific thermal diffusivities (Ref. 35). We broaden using a Lorentzian function with a width $\delta_{\omega, a v g}$.

${ }^{81}$ For the LJ alloys with $c \leq 0.15$, the predicted $k_{A F}$ is strongly system-size dependent, indicating this diverging behavior. For LJ argon alloys at $c=0.5$, the divergence with system size is small for the range of system size studied $\left(N_{0}=4\right.$ to $\left.N_{0}=12\right)$. For $N_{0}=12, k_{A F} / k_{G K}=0.93$ because the finite system-size limits the diffusivities of the lowest frequencies.

${ }^{82}$ N. Xu, V. Vitelli, M. Wyart, A. J. Liu, and S. R. Nagel, Phys. Rev. Lett. 102, 038001 (2009).

${ }^{83}$ P. Sheng and M. Zhou, Science 253, 539-542 (1991).

${ }^{84}$ E. S. Landry, M. I. Hussein, and A. J. H. McGaughey, Phys. Rev. B 77, 184302 (2008).

${ }^{85}$ D. P. Sellan, E. S. Landry, J. E. Turney, A. J. H. McGaughey, and C. H. Amon, Phys. Rev. B 81, 214305 (2010).

${ }^{86}$ K. Esfarjani and H. T. Stokes, Phys. Rev. B 77, 144112 (2008).

${ }^{87}$ L. Chaput, A. Togo, I. Tanaka, and G. Hug, Phys. Rev. B 84, 094302 (2011).

${ }^{88}$ R. Cheaito, J. C. Duda, T. E. Beechem, K. Hattar, J. F. Ihlefeld, D. L. Medlin, M. A. Rodriguez, M. J. Campion, E. S. Piekos, and P. E. Hopkins, Phys. Rev. Lett. 109, 195901 (2012).

${ }^{89}$ C. Kittel, Phys. Rev. 75, 972-974 (1949).

${ }^{90}$ A. J. H. McGaughey and J. M. Larkin, "Predicting phonon properties from equilibrium molecular dynamics simulations," Ann. Rev. Heat Transfer (to be published).

${ }^{91}$ L. Landau, E. Lifshitz, and L. Pitaevskii, Statistical Physics, Part 2 (Elsevier Science \& Technology Books, 1980), Vol. 9, P. 2.

${ }^{92}$ G. P. Srivastava, The Physics of Phonons (Adam Hilger, Bristol, 1990).

${ }^{93}$ A. Rajabpour and S. Volz, J. Appl. Phys. 108, 094324 (2010). 